\title{
Demanda e Desemprego no Médio Prazo`
}

\author{
- JORGE EdUARDo de CASTRO SOROMENHO*
}

- JAYLSON JAIR DA SILVEIRA**

\begin{abstract}
RESUMO
Apresentamos um modelo keynesiano no qual o conceito tradicional de equilíbrio do mercado de trabalho é substituído por um jogo evolucionário. O modelo permite concluir que, ao contrário da análise tradicional, a demanda agregada é crucial para determinar o nível de emprego de médio prazo. Mostramos que, ao alterarem a parametrização da dinâmica evolucionária, a política econômica e o gasto autônomo geram bifurcações, caracterizadas por mudanças no número de equilíbrios e/ou nas propriedades de estabilidade. $O$ desemprego passa a ser compreendido não como resultado de uma rigidez nominal ad hoc, mas sim como uma propriedade emergente da interação entre agentes que buscam a melhor estratégia de barganha salarial em um ambiente de racionalidade limitada.
\end{abstract}

Palavras-Chave

modelo keynesiano, desemprego, jogos evolucionários

\section{ABSTRACT}

In this article, we propose a keynesian model in which the traditional labor market equilibrium is replaced by an evolutionary game. In such a model the aggregate demand plays the crucial role in the determination of the medium-run equilibrium, differently from the traditional analysis. We show that economic policy and autonomous spending generate bifurcations, characterized by changes in the number of equilibria and/or in the stability properties. Thus, unemployment is not a result of an ad hoc nominal rigidity, but arises as a spontaneous outcome of an interaction process in which bounded rational agents grope for the best wage bargaining strategy.

\section{KEYWORDS}

Keynesian model, unemployment, evolutionary games

\author{
JEL Classification \\ EI2, C79
}

\footnotetext{
+ Agradecemos à professora Claudia Heller por seus comentários. Versões prévias deste trabalho foram apresentadas no Seminário Temático: Economia e Complexidade - COMPLEX e no XXVIII Encontro de Econometria. Somos gratos igualmente aos participantes desses encontros que comentaram o trabalho. Naturalmente, os erros remanescentes são de nossa exclusiva responsabilidade.

* Departamento de Economia/FEA-SP/USP - Av. Professor Luciano Gualberto, 908 - Cidade Universitária - São Paulo - SP. CEP 05508-0I0 - Tel: (II) 309I-5916. E-mail: jecs@usp.br.

* Departamento de Economia/FEA-RP/USP - Av. Bandeirantes 3900 - Monte Alegre - Ribeirão Preto - SP. CEP 14049-000 - Tel: (16) 3602-39I0. E-mail: jaylson@usp.br.

(Recebido em junho de 2006. Aceito para publicação em dezembro de 2007).
} 
Neste artigo, apresentamos um modelo keynesiano no qual o tratamento tradicional do mercado de trabalho é substituído por uma abordagem de jogo evolucionário. Essa abordagem é motivada pela conjectura de que o conceito de equilíbrio evolucionário ${ }^{1}$ constitui uma alternativa ao usual conceito de equilíbrio (excesso de demanda nulo) para discutir o desemprego involuntário num contexto dinâmico, sem apelar para a hipótese de rigidez nominal ad hoc.

Em uma economia com barganhas salariais descentralizadas, na qual há um mercado de trabalho interno a cada firma, os colegiados de trabalhadores negociam a cada período o salário nominal diretamente com os gestores das suas respectivas empresas, podendo aceitar a flexibilização do salário nominal ou exigir um específico salário nominal. Os payoffs (salários reais) dessas duas estratégias dependem não apenas do estado macroeconômico (nível geral de preços e taxa de desemprego), como ocorre na literatura tradicional, mas também das proporções em que as estratégias são adotadas na economia. A co-evolução da distribuição de estratégias dos colegiados de trabalhadores e do estado macroeconômico pode, a priori, não convergir para um estado em que todos os colegiados optam por flexibilizar o salário e, conseqüentemente, vigora o pleno emprego.

A teoria dos jogos evolucionários fornece instrumentos apropriados para analisar os possíveis comportamentos assintóticos (equilíbrios evolucionários) da dinâmica esboçada acima. No artigo, formalizaremos a evolução da distribuição de estratégias dos colegiados como uma dinâmica evolucionária em tempo contínuo, ${ }^{2}$ fundamentada na interpretação das escolhas das estratégias dos colegiados de trabalhadores ao longo do tempo como um processo de aprendizagem social em um ambiente de racionalidade limitada. ${ }^{3}$

1 O artigo insere-se na literatura que procura aplicar a teoria dos jogos evolucionários à análise de questões econômicas relevantes do ponto de vista teórico ou aplicado. Alguns trabalhos nacionais representativos dessa literatura são: Prado (1999 e 2001); Soromenho, Kadota e Prado (2001); Bonomo, Carrasco e Moreira (2003); Prado, Kadota e Soromenho (2003); Silveira (2003 e 2007); Silveira e Lima (2006); e Silveira e Sanson (2004).

2 No presente contexto, portanto, um jogo evolucionário é simplesmente uma dinâmica evolucionária em tempo contínuo, ou seja, um sistema de equações diferenciais ordinárias que: (i) satisfaz condiçôes mínimas de continuidade de modo que problemas de valor inicial possuam solução única; e (ii) cujas variáveis de estado são as distribuições das estratégias das populações de agentes em análise. Além disso, uma dinâmica evolucionária deve refletir um processo de seleção tal que, se uma estratégia propicia um payoff (salário real) melhor do que o da outra, a proporção com que ela é adotada na população cresce em termos relativos. Uma exposição e análise detalhada de dinâmicas evolucionárias em tempo contínuo podem ser encontradas em Ponti (2002).

3 Ponti (2002) resenha a literatura que modela dinâmicas evolucionárias por meio de equações diferenciais ordinárias no intuito de descrever o modo pelo qual agentes, num contexto de racionalidade limitada, ajustam suas escolhas a um ambiente em mudança. $\mathrm{O}$ autor interpreta as dinâmicas evolucionárias em tempo contínuo como modelos de aprendizagem, classificando-os em três grupos: modelos de aprendizagem individual, modelos de aprendizagem social e modelos de aprendizagem de crenças. No primeiro 
O artigo está organizado da seguinte forma: na seção 2 , discutimos brevemente a controvérsia sobre as razóes do desemprego keynesiano no intuito de justificar, de um ponto de vista metodológico, a adoção do conceito de equilíbrio evolucionário; na seção 3, adaptamos a estrutura típica dos modelos macroeconômicos de curto prazo, na qual historicamente se travou a controvérsia sobre o desemprego keynesiano, de modo que ela se torne compatível com o conceito de equilíbrio evolucionário; em seguida, na seção 4, discutimos as decisões dos trabalhadores e delas derivamos as equações dinâmicas do modelo; na seção 5 , analisamos as propriedades de médio prazo do modelo macroevolucionário. Investigamos a estrutura topológica do plano de fases da dinâmica evolucionária no intuito de demonstrar que políticas keynesianas de controle da demanda agregada podem não só deslocar a economia de uma bacia de atração para outra, mas também criar e destruir equilíbrios macroeconômicos. $\mathrm{Na}$ linguagem dos sistemas dinâmicos, demonstramos que as políticas keynesianas de controle da demanda agregada geram bifurcações, pois afetam a parametrização da dinâmica evolucionária.

\section{JUSTIFICATIVA METODOLÓGICA}

Na macroeconomia moderna, a demanda agregada tem um papel bastante limitado na determinação do nível de emprego. A ela atribuem-se somente efeitos de curto prazo. Assim, se bem administradas, políticas de controle da demanda podem apenas reforçar a tendência da economia a se auto-equilibrar na taxa natural de desemprego. Essa postura, quase consensual na profissão, é fruto da controvérsia que marcou a história da macroeconomia no século passado a respeito das causas do desemprego. No intuito de esclarecer a motivação do modelo apresentado neste artigo, convém recordar, ainda que de forma breve e impressionista, as principais etapas desse debate. ${ }^{4}$

A controvérsia que se seguiu à publicação da Teoria Geral (KEYNES, 1936) pode ser dividida em duas fases, que se distinguem pelos contextos metodológicos nos quais se travam as discussões. Na primeira fase, o contexto é eminentemente estático: a questão central é identificar qual seria a oferta de trabalho adequada à teoria de Keynes. Na segunda, o desemprego passa a ser concebido como uma situação de desequilíbrio em modelos dinâmicos.

tipo, sucessos e falhas do agente influenciam diretamente suas escolhas de estratégias. No segundo tipo, sucessos e falhas dos outros agentes afetam a probabilidade de escolhas de estratégias de cada agente. Finalmente, no terceiro tipo, a experimentação afeta somente as crenças.

4 Para uma apresentação da história do modelo IS-LM, ver Darity eYoung (1995). Os principais aspectos do debate foram resumidos por James Tobin em diversos textos (1993, 1994 e 1997). Os argumentos sinteticamente expostos nesta introdução são discutidos em detalhe em Soromenho (2003). 
O clássico artigo de Modigliani, de 1944, encerra a primeira fase. Modigliani propôs uma oferta de trabalho que, a depender dos valores de alguns parâmetros, representava o caso clássico ou o keynesiano. Na versão clássica, a quantidade ofertada de trabalho dependia do salário real. Na keynesiana, o salário nominal era rígido se a quantidade de trabalho fosse inferior à de pleno emprego; a partir desse nível, vigorava a oferta clássica. Em seguida, combinou essas ofertas com a IS e diferentes especificações do equilíbrio do mercado de ativos. Tornava-se possível não só sintetizar as discussões travadas até então em torno da Teoria Geral como também identificar a condição necessária e suficiente para a persistência do desemprego involuntário. Modigliani concluía que, com exceção dos casos da armadilha da liquidez e da perfeita inelasticidade da demanda agregada em relação aos juros, o desemprego involuntário exigia salários nominais rígidos. ${ }^{5}$

Todavia, quando a relevância da flexibilidade dos salários é discutida desse modo, tem-se a impressão de que o próprio contexto metodológico é inadequado. Com efeito, se, por um lado, não é razoável simplesmente postular a rigidez salarial, sem referência a razões que poderiam justificá-la, por outro, adotar a oferta clássica de trabalho também não constitui uma alternativa válida, visto que um sistema de equações que admite apenas um determinado valor como solução para uma variável endógena é simplesmente inconsistente se for suposto que essa variável assume outro valor. A análise estática não é o ambiente adequado, portanto, para responder à questão: 'o que acontece se o valor não for o de equilíbrio?', visto que essa possibilidade está descartada pelo próprio caráter do modelo. Na literatura moderna, a percepção dessa inadequação traduz-se na distinção entre flexibilidade e perfeita flexibilidade de salários. No segundo caso, diz-se que o desemprego é eliminado por definição (p. e. HAHN; SOLOW, 1997, p. 49) ou de modo quase tautológico (TOBIN, 1997, p. 20).

A segunda fase do debate decorre das discussões travadas em torno do efeito riqueza. Como se sabe, a introdução da riqueza na demanda agregada - os efeitos PigouHaberler-Patinkin - objetivaram, primordialmente, restaurar as propriedades de auto-equilibração das economias capitalistas no pleno emprego mesmo no caso da armadilha da liquidez. Porém, em decorrência da natureza dinâmica desses efeitos, a discussão deslocou-se para o contexto metodológico que se fazia necessário. Isto levou a uma nova percepção da natureza do desemprego, como relatou Harry Johnson:

5 De fato, por um lado, independentemente da especificação da demanda de moeda, a hipótese de salários rígidos permitia caracterizar o desemprego como situação de equilíbrio. Assim, essa hipótese era suficiente para garantir o desemprego. Por outro lado, se os salários fossem flexíveis não poderia existir desemprego. Logo, a rigidez salarial era condição necessária para a existência de subocupação da mãode-obra. A teoria da preferência pela liquidez era irrelevante. O desemprego involuntário devia-se única e exclusivamente à rigidez dos salários nominais. 
'unemployment equilibrium' has to be reinterpreted as a disequilibrium situation in which dynamic adjustment is proceeding very slowly; this is the interpretation of mathematical economists such as Leontief, Patinkin, and Clower, and is, I believe, a fair modern translation of Keynes's short period equilibrium technique. (JOHNSON, 1961, p. $12-13)$

Do ponto de vista teórico, no entanto, os efeitos da variação da riqueza no consumo não são isentos de ambigüidade. É necessário, como se sabe, distinguir entre riqueza interna e externa. Em relação à riqueza interna, a deflação pode favorecer credores, cuja propensão a consumir é menor do que a dos devedores, diminuindo, portanto, o consumo agregado. Restaria, então, como esperança de restaurar as propriedades de auto-equilibração no pleno emprego, o efeito da riqueza externa sobre o consumo. Sob o prisma empírico, o efeito riqueza revelou-se irrelevante. Segundo Tobin (1993, p. 87), o próprio Patinkin assinalou que, embora o valor da riqueza privada líquida tenha aumentado 46 por cento de 1929 a 1932, a renda real caiu 40 por cento. Apesar dessa evidência empírica, o efeito riqueza foi considerado por muitos um argumento teórico irrefutável em favor da tese clássica. Por que motivos? Podese, talvez, sintetizar essa tese da seguinte forma: se os salários continuassem a cair, em virtude do excesso de oferta de trabalho, os preços reduzir-se-iam, e, em algum momento, a riqueza real externa seria tão grande que, a despeito dos efeitos adversos relativos à riqueza interna, a demanda agregada deveria elevar-se, restaurando o pleno emprego. Pressuposto nesse raciocínio estava, evidentemente, a hipótese de que salários continuariam a cair, e com eles os preços, enquanto houvesse excesso de oferta no mercado de trabalho. Mas, se esse não fosse o caso, validar-se-ia novamente o argumento de que a condição suficiente e necessária era a rigidez nominal. De qualquer modo, prevalecia, então, a tese da centralidade da hipótese de rigidez nominal e a discussão deslocou-se para os motivos que poderiam justificá-la. Como se sabe, explicar a rigidez passou a constituir parte importante da agenda de pesquisas dos novos keynesianos.

Em suma, o brevíssimo resumo apresentado acima permite identificar um ponto importante para compreender a controvérsia. Na primeira fase, o contexto metodológico era inadequado para a discussão do tema: no âmbito estático, flexibilidade de salários só pode significar que o excesso de oferta no mercado de trabalho é nulo, i. e., vigora o pleno emprego por definição. Na segunda fase, o debate desenvolveu-se no contexto dinâmico que se fazia necessário, porém, a própria especificação dos modelos dinâmicos tinha por referência o conceito de equilíbrio clássico. Ou seja, supunha-se que a dinâmica de salários nominais podia ser descrita por uma equação diferencial por meio da qual se impunha que, se existisse excesso de oferta, o preço da mercadoria em questão deveria diminuir. Segue-se, então, que o único valor es- 
tacionário para o salário nominal é aquele para o qual o salário real implica excesso de oferta nulo. Pode ser que a economia convirja apenas de modo muito lento para esse valor estacionário, ou mesmo que haja inicialmente um aprofundamento da recessão, mas, aparentemente, o efeito riqueza garantiria que o resultado final seria o de pleno emprego.

Assim, embora muito tenha sido suposto a respeito da dinâmica global do sistema com base em análises que eram apenas locais, ${ }^{6}$ pode-se afirmar que equilíbrio de médio prazo e desemprego keynesiano eram incompatíveis. $\mathrm{O}$ máximo a que se podia aspirar era demonstrar que a estabilidade do único equilíbrio do modelo constituía apenas uma propriedade local e que a flexibilidade dos salários e preços poderia, na verdade, desestabilizar a economia, como havia sugerido o próprio Keynes. A adoção de imperfeições no mercado de trabalho pouco alterou esse quadro. Por exemplo, no conhecido livro-texto de Blanchard (1999), flexibilidade imperfeita de salários e expectativas adaptativas são suficientes para assegurar que, no médio prazo, vigora uma taxa natural de desemprego redefinida de modo a ser compatível com a presença de sindicatos. As políticas de controle de demanda revelam-se, então, ineficazes para aumentar permanentemente o nível de emprego. Coerentemente, a macroeconomia de inspiração keynesiana passou a ser organizada em torno do conceito de taxa natural de desemprego, e as razôes do desemprego persistente foram atribuídas aos determinantes dessa taxa.

Diante desses resultados, cabe perguntar, no entanto, se esse conceito de equilíbrio - que orienta a especificação da própria equação dinâmica e que exclui o desemprego keynesiano como um possível estado estacionário para o qual possa convergir a dinâmica macroeconômica - é conveniente para discutir o fenômeno em questão. De um ponto de vista metodológico, talvez se revele proveitoso conceber um modelo no qual o desemprego keynesiano e o pleno emprego sejam ambos estados estacionários e, em seguida, examinar em que circunstâncias a economia converge para um ou outro equilíbrio. Evidentemente, isto pressupõe a redefinição do próprio conceito de equilíbrio.

A conjectura que orienta este artigo é a de que o conceito de equilíbrio evolucionário pode vir a se revelar uma alternativa ao conceito tradicional para apreender a idéia de desemprego involuntário num contexto dinâmico. Dois motivos concorrem para isso. Primeiro, modelos de jogos evolucionários apresentam, normalmente, múltiplos equilíbrios. Abre-se o espaço, portanto, para conceber estados de equilíbrio de médio prazo compatíveis com desemprego e, conseqüentemente, restaurar a importância das políticas fiscal e monetária como determinantes do nível de ocupação da mão-de-obra. Segundo, nos modelos evolucionários, os equilíbrios são selecionados

6 Ver, a respeito, Flasche, Reiner e Semmler (1997). 
por meio de dinâmicas que podem ser microfundadas de diferentes formas, que vão desde comportamentos de imitação simples a processos de aprendizagem social mais complexos (PONTI, 2002, p. 189). Isto permite superar as objeções justamente feitas aos processos de tâtonnement sintetizadas, há muitos anos, por Koopmans:

The various assumptions that have been used to describe the adjustment of price or quantity in a commodity market clearly show their parentage in the laws of the physical sciences. If, for instante, the net rate of increase in price is assumed to be proportional to the excess of demand over supply, whose behavior is thereby expressed? And how is that behavior motivated? (KOOPMANS, 1957, p. 179)

A utilização desse conceito de equilíbrio permite, portanto, redefinir a flexibilidade de salários que, no contexto evolucionário, significa permitir que os agentes escolham, conforme suas conveniências explicitamente modeladas, flexibilizar o salário ou não. Em síntese, a adoção de uma estratégia de formalização que comporta multiplicidade de equilíbrios e fundamenta a dinâmica em interações individuais abre a possibilidade de compreender o desemprego - e, eventualmente, outras regularidades macroeconômicas - como uma propriedade emergente.

\subsection{O Modelo}

Considere uma economia composta de $h$ firmas maximizadoras de lucro, que produzem um único bem $Y$, e $N$ trabalhadores $(N>h)$. O estoque de capital é constante e a produção de cada firma $j$ é uma função, $f: R_{+} \rightarrow R_{+}$, do número de trabalhadores empregados, $N_{j}$. Além das hipóteses usuais $f^{\prime} \geq 0$ e $f^{\prime \prime}<0$, supomos que o produto e a produtividade marginal sejam limitados:

$$
f(0)=0 ; \sup f(\cdot)=b<\infty ; 0<f^{\prime}(0)=a<\infty ; \text { e } \lim _{N_{j} \rightarrow \infty} f^{\prime}\left(N_{j}\right)=0
$$

para qualquer firma $j=1,2, \ldots, h$.

Admita que exista um mercado de trabalho interno a cada firma no qual se negociam salários nominais no início do período de produção. Essas negociações dão-se entre 
os gestores da empresa e o colegiado de trabalhadores. Os colegiados podem adotar apenas duas estratégias $(i=1,2)$ : flexibilizam o salário nominal, $w_{1}$, ou exigem um específico salário nominal fixo $w_{2}$. As firmas podem ser divididas, então, em dois grupos ou setores conforme a estratégia adotada por seus trabalhadores. $\mathrm{O}$ setor 1 será denominado clássico ou flexível, e o setor 2 será dito keynesiano ou rígido. Sejam $N_{i}$ e $h_{i}$ o número de trabalhadores e o de firmas do tipo $i$ que compõem o setor $i$. Dentro de cada grupo, supomos que a firmas sejam perfeitamente homogêneas, isto é, empregam o mesmo número de trabalhadores e pagam salários nominais iguais, ou seja, $N_{j}=N_{i} / h_{i}$ e $w_{j}=w_{i}$ para toda firma $j=1,2, \ldots, h_{i}$ do tipo $i=1,2$.

No setor clássico, supomos que os trabalhadores, ao flexibilizarem seus salários, garantam a manutenção dos seus empregos, mas sujeitem-se a um salário real $\omega_{1}$ que depende do total de trabalhadores que aceitam $w_{1}$. Ou seja, $N_{1}$ compreende não só os trabalhadores que participaram das negociações salariais no início do período, mas também quaisquer outros que aceitem aquele salário nominal. $\mathrm{O}$ processo pelo qual se determina esse salário nominal é deixado em aberto. Assumimos, simplesmente, que ele opera como no tradicional modelo clássico. Assim, $N_{1}$ é a quantidade ofertada de trabalho nesse setor e o salário real $\omega_{1}$ é igual à produtividade marginal $f^{\prime}\left(N_{1} / h_{1}\right)$.

No setor keynesiano, os trabalhadores podem perder seus postos, pois nada assegura que a maximização de lucro induza as firmas a empregarem todos os que optaram por essa estratégia. Assim, $\omega_{2} \equiv w_{2} / p=f^{\prime}\left(N_{2} / h_{2}\right)$, mas, ao contrário do que ocorre no primeiro caso, o nível de emprego efetivo nesse setor, $N_{2}$, pode ser menor do que o número de trabalhadores participantes das negociações internas.

Para completar a descrição das populações, seja $U$ o número de desempregados. Por construção, um trabalhador desempregado, ou já estava nessa situação no período anterior, e decidiu não procurar trabalho no setor de salários flexíveis na transição entre períodos, ou estava empregado no setor de salários rígidos e perdeu a sua ocupação no atual período.

Considere as seguintes definições: $n_{1}=N_{1} / N ; n_{2}=N_{2} / N ; u=U / N$; $\alpha=h_{1} / h$; e $c=N / h .^{7} \mathrm{O}$ número de trabalhadores empregados em cada tipo de firma pode ser expresso, então, em termos dessas participações das populações de trabalhadores e firmas: $N_{1} / h_{1}=c \quad n_{1} / \alpha$ e $N_{2} / h_{2}=c \quad n_{2} /(1-\alpha)$.

7 A restrição natural dessa economia é, evidentemente: $n_{1}+n_{2}+u=1$. 
Como o produto do setor $i$ é igual ao número de empresas do tipo $i$ multiplicado pela produção por firma, $h_{i} f(\cdot)$, as produções de uma firma do tipo 1 e de uma do tipo 2, normalizadas pelo número total de firmas da economia, são, respectivamente:

$$
y_{1}=\alpha f\left(\frac{c n_{1}}{\alpha}\right) ; \quad \text { e } \quad y_{2}=(1-\alpha) f\left(\frac{c n_{2}}{1-\alpha}\right)
$$

No intuito de identificar o domínio dessas funções de produção, considere os seguintes conjuntos:

$$
\Omega_{i}=\left\{\left(\alpha, n_{i}\right) \in R^{2}: 0 \leq \alpha \leq 1,0 \leq n_{i} \leq 1\right\}, \quad i=1,2
$$

Visto que a existência de firmas em um setor depende da presença de trabalhadores nesse mesmo setor, se $\alpha=0 \quad(\alpha=1)$, então, $n_{1}\left(n_{2}\right)$ também deve ser nulo. Assim, na nossa economia, os vetores $\left(\alpha, n_{i}\right)$ economicamente relevantes devem pertencer ao conjunto int $\Omega_{i} \cup\{(0,0),(1,1)\}$. Não obstante, a análise é facilitada se definirmos as funções de produção nos conjuntos $\Omega_{i}$. Nesse sentido, observe que se $\alpha=0$, a produção do setor 1 é uma indeterminação matemática, e o mesmo ocorre para o setor 2 quando $\alpha=1$. No entanto, para $i=1,2$ e qualquer $\left(\alpha, n_{i}\right) \in \Omega_{i}$, tal que $i-1-\alpha \neq 0$ e $\bar{n}_{i} \in[0,1]$, temos que:

$$
\lim _{\left(\alpha, n_{1}\right) \rightarrow\left(0, \bar{n}_{1}\right)} \alpha f\left(\frac{c n_{1}}{\alpha}\right)=0 ; \mathrm{e} \quad \lim _{\left(\alpha, n_{2}\right) \rightarrow\left(1, \bar{n}_{2}\right)}(1-\alpha) f\left(\frac{c n_{2}}{1-\alpha}\right)=0
$$

Com efeito, seja a seqüência $x_{1}^{s}=\left(\alpha^{s}, n_{1}^{s}\right) \in \Omega_{1}, \alpha^{s} \neq 0$ e $s \in N$. Como, por hipótese, $f(0)=0$ e $\sup f=b$, existe uma vizinhança em torno de $\left(0, \bar{n}_{1}\right)$ para a qual $f\left(c n_{1}^{s} / \alpha^{s}\right) \in[0, b]$ quando $\lim _{s \rightarrow \infty} x_{1}^{s}=\left(0, \bar{n}_{1}\right)$. Logo, $\lim _{s \rightarrow \infty} \alpha^{s} f\left(\begin{array}{ll}c & n_{1}^{s} / \alpha^{s}\end{array}\right)=0$ para qualquer sequiência $x_{1}^{s}$ tal que $\lim _{s \rightarrow \infty} x_{1}^{s}=\left(0, \bar{n}_{1}\right)$. Argumento análogo vale para o segundo limite.

Podemos, então, definir as seguintes funções contínuas em $\Omega_{1}$ e $\Omega_{2}$ : 


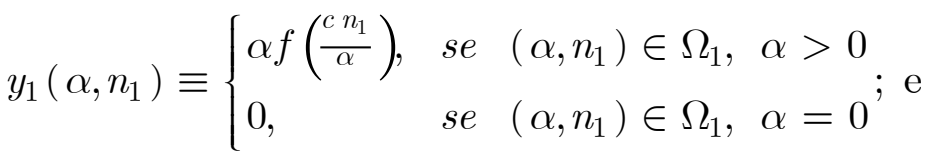

$$
\begin{aligned}
& y_{2}\left(\alpha, n_{2}\right) \equiv\left\{\begin{array}{lll}
(1-\alpha) f\left(\frac{c n_{2}}{1-\alpha}\right), & \text { se } & \left(\alpha, n_{2}\right) \in \Omega_{2}, \quad \alpha<1 \\
0, & \text { se } & \left(\alpha, n_{2}\right) \in \Omega_{2}, \quad \alpha=1
\end{array}\right.
\end{aligned}
$$

A produção agregada máxima da economia depende não apenas da quantidade total de trabalhadores, mas também - em decorrência da hipótese de rendimentos decrescentes - da distribuição de trabalhadores e empresas entre os dois setores. Se inexiste desemprego, $u=0$, ou seja, $n_{2}=1-n_{1}$, então a produção agregada de pleno emprego normalizada pelo número total de firmas é:

$$
y\left(\alpha, n_{1}\right)=y_{1}\left(\alpha, n_{1}\right)+y_{2}\left(\alpha, 1-n_{1}\right)
$$

Esta função é de classe $C^{2}$ e côncava em int $\Omega_{1}$. Esta última propriedade decorre do fato de que no conjunto aberto e convexo int $\Omega_{1}$ a matriz Hessiana $D^{2} y\left(\alpha, n_{1}\right)$ da função $y\left(\alpha, n_{1}\right)$ é negativa semidefinida para todo $\left(\alpha, n_{1}\right) \in$ int $\Omega_{1}$, pois os sinais dos menores principais são:

$$
\begin{aligned}
& \frac{\partial^{2} y}{\partial \alpha^{2}}=\frac{c^{2} n_{1}^{2} f^{\prime \prime}\left(\frac{c n_{1}}{\alpha}\right)}{\alpha^{3}}+\frac{c^{2}\left(1-n_{1}\right)^{2} f^{\prime \prime}\left(\frac{c\left(1-n_{1}\right)}{1-\alpha}\right)}{(1-\alpha)^{3}}<0 \\
& \frac{\partial^{2} y}{\partial n_{1}^{2}}=\frac{c^{2} f^{\prime \prime}\left(\frac{c n_{1}}{\alpha}\right)}{\alpha}+\frac{c^{2} f^{\prime \prime}\left(\frac{c\left(1-n_{1}\right)}{1-\alpha}\right)}{1-\alpha}<0 ; \mathrm{e} \\
& \left|D^{2} y\left(\alpha, n_{1}\right)\right|=-\frac{c^{4}\left(n_{1}-\alpha\right)^{2} f^{\prime \prime}\left(\frac{c\left(1-n_{1}\right)}{1-\alpha}\right) f^{\prime \prime}\left(\frac{c n_{1}}{\alpha}\right)}{(1-\alpha)^{3} \alpha^{3}} \geq 0 .
\end{aligned}
$$

Observe que se $\alpha=n_{1},\left|D^{2} y\left(\alpha, n_{1}\right)\right|=0$. Dada a concavidade da função $y\left(\alpha, n_{1}\right)$ em int $\Omega_{1}$, a condição necessária e suficiente para um máximo em int $\Omega_{1}$ é $D y\left(\alpha, n_{1}\right)=0$, ou seja: 


$$
\begin{gathered}
\frac{\partial y}{\partial n_{1}}=c f^{\prime}\left(\frac{c n_{1}}{\alpha}\right)-c f^{\prime}\left(\frac{c\left(1-n_{1}\right)}{1-\alpha}\right)=0 ; \mathrm{e} \\
\frac{\partial y}{\partial \alpha}=f\left(\frac{c n_{1}}{\alpha}\right)-\frac{c n_{1}}{\alpha} f^{\prime}\left(\frac{c n_{1}}{\alpha}\right) \\
-f\left(\frac{c\left(1-n_{1}\right)}{1-\alpha}\right)+\frac{c\left(1-n_{1}\right)}{1-\alpha} f^{\prime}\left(\frac{c\left(1-n_{1}\right)}{1-\alpha}\right)=0
\end{gathered}
$$

É fácil constatar que qualquer $\alpha$ igual a $n_{1}$ resolve o sistema e, portanto, o produto máximo é $y\left(\alpha, n_{1}\right)=\alpha f(c)+(1-\alpha) f(c)=f(c)$. O resultado mantém-se ao considerarmos o domínio fechado $\Omega_{1}$, pois $y\left(\alpha, n_{1}\right)$ é contínua, visto que é soma de funções contínuas, e, se $\alpha=n_{1}$ e um dos setores é extinto, o produto máximo também é igual a $f(c)$.

As remunerações pagas nos setores são iguais às respectivas produtividades marginais:

$$
\omega_{1}=f^{\prime}\left(\frac{c n_{1}}{\alpha}\right) ; \omega_{2}=f^{\prime}\left(\frac{c n_{2}}{1-\alpha}\right)
$$

A produtividade marginal das firmas do tipo $2, f^{\prime}\left(c n_{2} /(1-\alpha)\right)$, é função continuamente diferenciável e estritamente decrescente no intervalo $[0, \infty)$. Definindo $g\left(\omega_{2}\right)$ como a inversa da segunda equação de (3.13), temos:

$$
n_{2}=\frac{1-\alpha}{c} g\left(\omega_{2}\right), \omega_{2} \in(0, a] ; g^{\prime}\left(\omega_{2}\right)=1 / f^{\prime \prime}\left(\frac{c n_{2}}{1-\alpha}\right)<0
$$

Substituindo em (3.6), definimos o produto do setor por firma como função de $\omega_{2}$ :

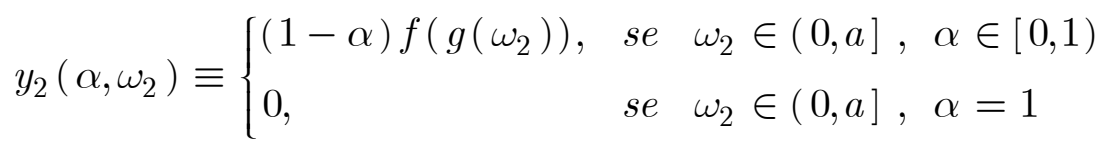

Observe que essa função é contínua no domínio especificado, pois como $f\left(g\left(\omega_{2}\right)\right)$ é limitada, para qualquer seqüência $x^{s}=\left(\alpha^{s}, \omega_{2}^{s}\right) \in\left\{\left(\alpha, \omega_{2}\right) \in R_{+}^{2}: 0<\omega_{2} \leq\right.$ 
$\leq a, 0 \leq \alpha<1\}, s \in N$, tal que $\lim _{s \rightarrow \infty} x^{s}=\left(1, \bar{\omega}_{2}\right), \bar{\omega}_{2} \in(0, a]$, o limite de $\left(1-\alpha^{s}\right) f\left(g\left(\omega_{2}^{s}\right)\right)$ é igual a zero.

Para manter o modelo o mais simples possível, admita que a demanda agregada $y^{d}$ (igualmente normalizada pelo número total de firmas) depende negativamente do nível de preços $p$ (como forma sintética de incorporar o efeito riqueza e o efeito Keynes) e positivamente de parâmetro $A \in R_{+}$, que resume todos os outros elementos que possam influenciar o dispêndio global. Como $p=w_{2} / \omega_{2}$, aumentos do salário real do setor rígido elevam o dispêndio agregado. Supomos, ademais, que se o preço tende a infinito, a demanda é nula, sendo positiva nos demais casos. Seja, então, $y^{d}=D\left(\omega_{2}, A\right)$, tal que:

$D(0, A)=0 ; D\left(\omega_{2}, A\right) \geq 0$ para $\omega_{2}>0 ; D_{1} \equiv \frac{\partial D}{\partial \omega_{2}}>0 ;$ e $D_{2} \equiv \frac{\partial D}{\partial A}>0$.

Admita, além disso, como hipótese meramente conveniente de um ponto de vista técnico, que a demanda seja maior ou igual ao produto máximo sempre que $\omega_{2}$ assume seu valor máximo, ou seja:

$$
D(a, A) \geq f(c)
$$

Por fim, suponha que o mercado de produto esteja sempre em equilíbrio. No curto prazo, o modelo resume-se às seguintes equações:

$$
\begin{aligned}
& \omega_{1}=f^{\prime}\left(\frac{c n_{1}}{\alpha}\right) \\
& n_{2}=\frac{1-\alpha}{c} g\left(\omega_{2}\right) ; \mathrm{e} \\
& D\left(\omega_{2}, A\right)-y_{1}\left(\alpha, n_{1}\right)-y_{2}\left(\alpha, \omega_{2}\right)=0 .
\end{aligned}
$$

Após terem sido realizadas as negociações, na transição entre períodos, o número de firmas em cada grupo e o de trabalhadores que se dispóem a trabalhar ao salário flexível estão determinados. Assim, no curto prazo $\alpha$ e $n_{1}$ são variáveis exógenas. O modelo compreende, então, três equações e três incógnitas, $\omega_{1}, \omega_{2}$ e $n_{2}$. A condição de maximização de lucro (3.18) das firmas do tipo l é suficiente para deter- 
minar o salário real do setor flexível $\omega_{1}^{*}$, o qual independe, portanto, do parâmetro $A$. A condição de equilíbrio no mercado de bens (3.20) determina o salário real do setor rígido $\omega_{2}^{*}$ e, identificado este, a condição de maximização de lucro (3.19) das firmas do tipo 2 determina o nível de emprego do setor keynesiano $n_{2}^{*}$.

\subsection{Os Equilibrios do Curto Prazo}

Para atender aos nossos propósitos, o modelo deve compreender três tipos de equilíbrios associados a diferentes valores dos parâmetros $\alpha$ e $n_{1}$. Primeiro, um equilíbrio keynesiano puro que se caracteriza pela existência apenas do setor rígido: $\left(\alpha, n_{1}\right)=(0,0)$. Segundo, um equilíbrio, que denominaremos estratégia mista, no qual os dois setores da economia coexistem: $\left(\alpha, n_{1}\right) \in$ int $\Omega_{1}$. Por último, um equilíbrio clássico puro que se caracteriza pela existência apenas do setor flexível, no qual todos os salários são perfeitamente flexíveis e o pleno emprego vigora por definição: $\left(\alpha, n_{1}\right)=(1,1)$. A seguir, discutimos como o modelo comporta esses três casos.

Considere a função de excesso de demanda do mercado de produto:

$$
E\left(\omega_{2}, \alpha, n_{1}, A\right)=D\left(\omega_{2}, A\right)-y_{1}\left(\alpha, n_{1}\right)-y_{2}\left(\alpha, \omega_{2}\right)
$$

Mostramos, a seguir, que a solução $\omega_{2}^{*}$ que elimina o excesso de demanda é função de $\alpha, n_{1}$ e de $A$.

Proposição 3.1. Existe uma função $\phi: \Omega_{1} \times R_{+} \rightarrow(0, a]$, tal que,

$$
\omega_{2}^{*}=\phi\left(\alpha, n_{1}, A\right)
$$

e $E\left(\omega_{2}^{*}, \alpha, n_{1}, A\right)=0$, para todo $\left(\alpha, n_{1}, A\right) \in \Omega_{1} \times R_{+}$.

Prova: Inicialmente, utilizamos o teorema do valor intermediário (TVI) no intuito de garantir a existência de solução de (3.21). Nesse sentido, observe que $E(\cdot)$ é contínua no domínio $(0, a] \times \Omega_{1} \times R_{+}$, pois é soma de funções contínuas. De $(3.15)$ e (3.16) segue que

$$
\lim _{\omega_{2} \rightarrow 0} E\left(\omega_{2}, \alpha, n_{1}, A\right)=-\alpha f\left(c \quad n_{1} / \alpha\right)-(1-\alpha) b<0
$$


Como o produto máximo é $f(c)$, a hipótese (3.17) é suficiente para assegurar que, quando $\omega_{2}$ assume seu valor máximo, $a$,

$$
E\left(a, \alpha, n_{1}, A\right)=D(a, A)-\alpha f\left(c \quad n_{1} / \alpha\right) \geq f(c)-\alpha f\left(c \quad n_{1} / \alpha\right) \geq 0
$$

Logo, pelo TVI existe um $\omega_{2}^{*} \in(0, a]$ tal que $E\left(\omega_{2}^{*}, \alpha, n_{1}, A\right)=0$. Para provar a unicidade da solução, observe que, para todo $\omega_{2} \in(0, a]$, a função de excesso de demanda é estritamente crescente com relação ao salário real no setor 2 , pois, dada a definição (3.15) e recordando que $g^{\prime}\left(\omega_{2}\right)<0$,

$$
\frac{\partial E}{\partial \omega_{2}}=D_{1}-(1-\alpha) f^{\prime}\left(g\left(\omega_{2}\right)\right) g^{\prime}\left(\omega_{2}\right)=D_{1}-(1-\alpha) \omega_{2} g^{\prime}\left(\omega_{2}\right)>0 .
$$

Assim, para cada vetor $\left(\alpha, n_{1}, A\right) \in \Omega_{1} \times R_{+}$existe um único $\omega_{2}^{*}$ ao qual o excesso de demanda é nulo.

Como a função de excesso de demanda (3.21) possui derivadas parciais contínuas para todo $\left(\alpha, n_{1}, A\right) \in \Omega_{1} \times R_{+}$tal que $\alpha \neq 0$, segue-se, pelo teorema da função implícita, que $\phi\left(\alpha, n_{1}, A\right)$ é de classe $C^{1}$ para todo $\left(\alpha, n_{1}, A\right) \in \Omega_{1} \times R_{+}$tal que $\alpha \neq 0$. No apêndice demonstramos que a continuidade dessa função se estende a pontos $\left(\alpha, n_{1}, A\right)$ do domínio tais que $\alpha=0$.

Substituindo $\omega_{2}^{*}$ em (3.19), identificamos o nível de emprego de equilíbrio de curto prazo:

$$
n_{2}^{*}=\frac{1-\alpha}{c} g\left(\phi\left(\alpha, n_{1}, A\right)\right)
$$

Em suma, para cada vetor $\left(\alpha, n_{1}, A\right) \in \Omega_{1} \times R_{+}$, pelas equações (3.18), (3.22) e (3.26) determina-se um único vetor $\left(\omega_{1}^{*}, \omega_{2}^{*}, n_{2}^{*}\right)$ que define o equilíbrio de curto prazo.

Os seguintes resultados de análise estática comparativa serão utilizados na análise do médio prazo: 


$$
\begin{aligned}
& \phi_{\alpha} \equiv \frac{\partial \omega_{2}^{*}}{\partial \alpha}=\frac{f\left(\frac{c n_{1}}{\alpha}\right)-\frac{c n_{1}}{\alpha} f^{\prime}\left(\frac{c n_{1}}{\alpha}\right)-f\left(g\left(\omega_{2}^{*}\right)\right)}{D_{1}-(1-\alpha) \omega_{2}^{*} g^{\prime}\left(\omega_{2}^{*}\right)} \\
& \frac{\partial n_{2}^{*}}{\partial \alpha}=\frac{1}{c}\left[(1-\alpha) g^{\prime}\left(\omega_{2}^{*}\right) \phi_{\alpha}-g\left(\omega_{2}^{*}\right)\right] \\
& \phi_{n_{1}} \equiv \frac{\partial \omega_{2}^{*}}{\partial n_{1}}=\frac{c f^{\prime}\left(\frac{c n_{1}}{\alpha}\right)}{D_{1}-(1-\alpha) \omega_{2}^{*} g^{\prime}\left(\omega_{2}^{*}\right)}>0 ; \\
& \frac{\partial n_{2}^{*}}{\partial n_{1}}=\frac{1-\alpha}{c} g^{\prime}\left(\omega_{2}^{*}\right) \phi_{n_{1}}<0 ; \\
& \phi_{A} \equiv \frac{\partial \omega_{2}^{*}}{\partial A}=-\frac{D_{2}}{D_{1}-(1-\alpha) \omega_{2}^{*} g^{\prime}\left(\omega_{2}^{*}\right)}<0 ; \mathrm{e} \\
& \frac{\partial n_{2}^{*}}{\partial A}=\frac{1-\alpha}{c} g^{\prime}\left(\omega_{2}^{*}\right) \phi_{A}>0
\end{aligned}
$$

As soluções obtidas para o curto prazo requerem algumas observações. Primeiro, o equilíbrio keynesiano puro exige que $\alpha$ seja nulo. Neste caso particular, a função de excesso de demanda é $E\left(\omega_{2}, 0, n_{1}, A\right)=D\left(\omega_{2}, A\right)-f\left(g\left(\omega_{2}\right)\right)$, que não depende de $n_{1}$. Ou seja, se $\alpha=0$, a função $\phi\left(\alpha, n_{1}, A\right)$ é constante em relação a $n_{1}$. Evidentemente, do ponto de vista econômico, o equilíbrio keynesiano exige que $n_{1}$ também seja igual a zero. Todavia, identificar a solução de (3.21), destacando que $\phi_{n_{1}}\left(0, n_{1}, A\right)=0$ para qualquer $n_{1}$, é conveniente para demonstrações posteriores. Segundo, o equilíbrio clássico puro verifica-se quando $\left(\alpha, n_{1}\right)=(1,1)$. A solução matemática $\omega_{2}^{*}=\phi(1,1, A)>0$ é, evidentemente, desprovida de significado econômico, visto que o setor 2 está extinto. Não obstante, como $\omega_{2}^{*}$ é estritamente positivo e $g(\cdot)$ é contínua, $g\left(\omega_{2}^{*}\right)$ assume valores finitos, o que é suficiente para garantir que $n_{2}^{*}=0 \quad g(\phi(1,1, A))=0$. O modelo comporta, portanto, uma solução clássica consistente com a extinção do setor rígido. ${ }^{8}$

8 As soluções matemáticas nos casos keynesiano puro e de estratégia mista compreendem casos que violam a restrição natural, $n_{1}+n_{2} \leq 1$. Considere, por exemplo, o caso keynesiano puro. A demanda é monotonicamente crescente em relação ao salário real do setor 2; a oferta é função monotonicamente decrescente de $\omega_{2}$ e, por definição, assume valores em $[0, b]$. Mas, se $\alpha=0$ e $n_{2}<1$, o produto máximo é $f(c)<b$. Abre-se a possibilidade, portanto, de que, para valores de $A$ suficientemente elevados, inexista equilíbrio keynesiano puro com significado econômico. 


\section{A TRANSIÇÃO ENTRE PERÍODOS}

\subsection{Fluxos Populacionais}

$\mathrm{Na}$ transição entre períodos, ocorrem as negociações. Firmas e trabalhadores podem mudar, então, seu tipo. Por um lado, empresas do setor de salários rígidos podem passar a pertencer ao grupo clássico, em virtude de uma mudança de atitude de seus trabalhadores, que passam a aceitar o salário $w_{1}$. Por outro, nas empresas do setor flexível, os trabalhadores podem exigir o salário nominal $w_{2}$, o que transforma essas empresas em firmas do setor keynesiano.

Considere o tempo uma variável contínua. Seja $\dot{h}_{i}=d h_{i} / d t$ a taxa de variação líquida de firmas no setor $i$. Essa taxa pode ser vista como resultado da diferença das taxas de influxo e efluxo, que denominaremos $\dot{h}_{i}^{i}$ e $\dot{h}_{i}^{e}$, respectivamente. Assim, temos $\dot{h}_{1}=\dot{h}_{1}^{i}-\dot{h}_{1}^{e}$; e $\dot{h}_{2}=\dot{h}_{2}^{i}-\dot{h}_{2}^{e}$. Como o total de firmas é dado, o acréscimo de uma das populações é exatamente igual ao decréscimo da outra, ou seja, $\dot{h}_{1}^{i}=\dot{h}_{2}^{e}$ e $\dot{h}_{1}^{e}=\dot{h}_{2}^{i}$. Recordando que $\alpha=h_{1} / h$, a variação do percentual de firmas no setor flexível é:

$$
\dot{\alpha}=\frac{\dot{h_{1}}}{h}=\frac{\dot{h}_{1}^{i}-\dot{h}_{1}^{e}}{h}
$$

Ao fluxo de firmas de 1 para 2 está associado um efluxo de trabalhadores do setor 1, $\dot{N}_{1}^{e}$, que é igual ao número de firmas que saíram vezes o número de trabalhadores que cada firma empregava nesse setor.

$$
\dot{N}_{1}^{e}=\dot{h}_{1}^{e} \frac{N_{1}}{h_{1}}
$$

O influxo de trabalhadores para $1, \dot{N}_{1}^{i}$, é um pouco mais complicado. Uma parte desse influxo, $\dot{N}_{1}^{i 1}$, advém da mudança de firmas 2 para 1 e é igual ao número de firmas que saíram do setor 2 vezes o número de seus empregados:

$$
\dot{N}_{1}^{i 1}=\dot{h}_{2}^{e} \frac{N_{2}}{h_{2}}=\dot{h}_{1}^{i} \frac{N_{2}}{h_{2}}
$$


Um segundo influxo resulta dos desempregados do período anterior que decidem procurar emprego no setor 1 . Esse número não é necessariamente igual ao total de desempregados, pois parte deles persiste em procurar emprego no setor 2. Seja esse influxo designado por $\dot{N}_{1}^{i 2}$. Segue, então, que:

$$
\dot{N}_{1}=\dot{N}_{1}^{i}-\dot{N}_{1}^{e}=\dot{N}_{1}^{i 1}+\dot{N}_{1}^{i 2}-\dot{N}_{1}^{e}=\dot{h}_{1}^{i} \frac{N_{2}}{h_{2}}+\dot{N}_{1}^{i 2}-\dot{h}_{1}^{e} \frac{N_{1}}{h_{1}}
$$

Como $n_{1}=N_{1} / N$, sendo $N$ dado, temos:

$$
\dot{n}_{1}=\frac{\dot{N}_{1}}{N}=n_{2} \frac{\dot{h}_{1}^{i}}{h_{2}}+\frac{\dot{N}_{1}^{i 2}}{N}-n_{1} \frac{\dot{h}_{1}^{e}}{h_{1}}
$$

A Figura 1 ilustra os fluxos discutidos nesta seção.

FIGURA 1 - FLUXOS POPULACIONAIS
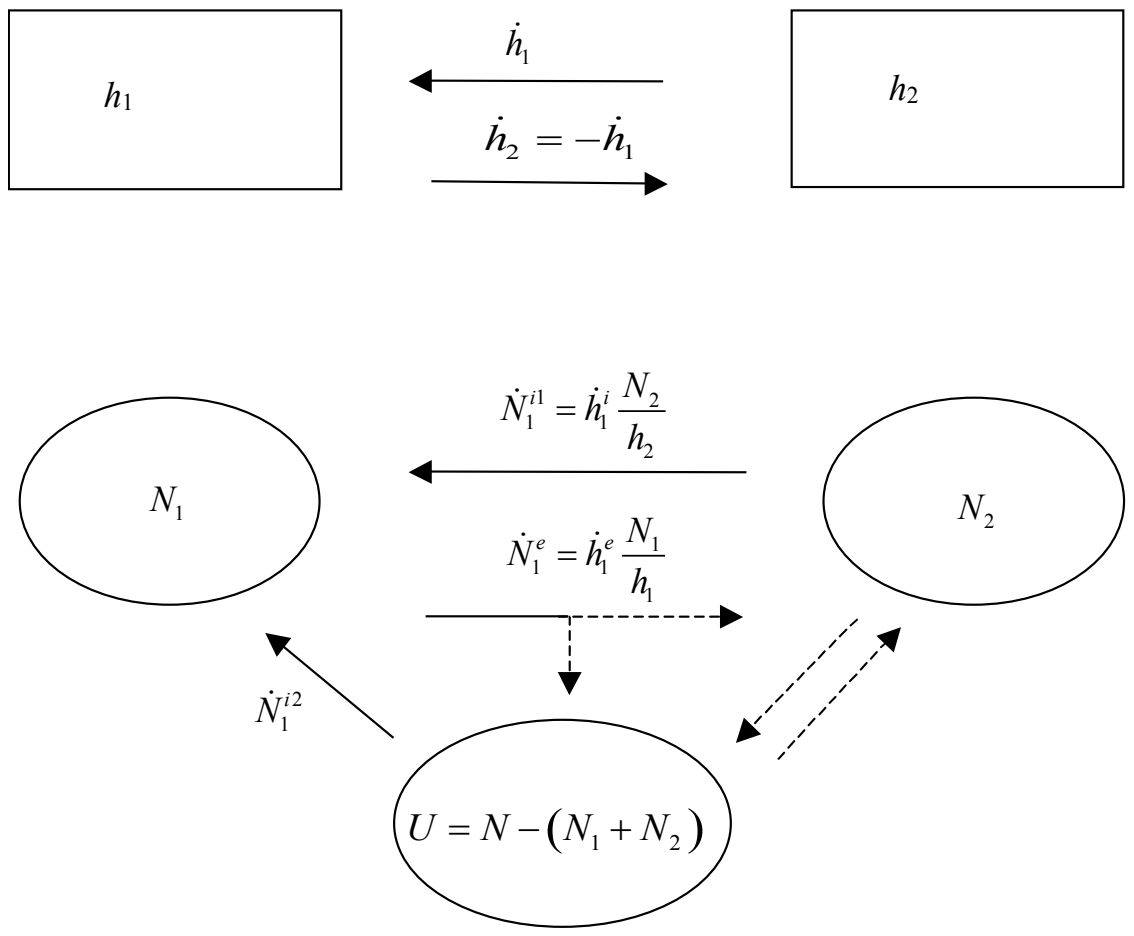

$\underset{----- \text { Determinado na transição entre períodos. }}{ }$ Determinado no equilíbrio de curto prazo. 


\subsection{As Decisões dos Trabalhadores}

Os fluxos das populações entre diferentes estratégias podem ser obtidos definindose as taxas de revisão das estratégias por unidade de tempo e as probabilidades de escolha dos agentes revisores (WEIBULL, 1995, p. 149). ${ }^{9}$ No nosso modelo, a estratégia dos trabalhadores empregados é decidida pelo conjunto, colegiado ou representação sindical dos trabalhadores de cada firma. Há, então, tantas unidades de decisão (ou agentes) quantas são as empresas dos dois setores da economia, i. e., $h_{1}$ e $h_{2}$. Já as estratégias dos trabalhadores desempregados são estritamente individuais. Como todos os agentes reavaliam suas estratégias na transição entre períodos, as taxas de revisão por unidade de tempo são iguais a um. Resta, então, determinar as probabilidades de escolha.

Considere, inicialmente, as decisões dos colegiados de trabalhadores empregados. Admita que esses agentes comparam os seus resultados (payoffs), os quais definiremos posteriormente, com os obtidos pelos trabalhadores de outra empresa escolhida aleatoriamente, segundo uma distribuição de probabilidade uniforme. A probabilidade de comparação ocorrer com uma empresa na qual vigorou a estratégia alternativa é igual $h_{1} / h$, para os agentes do setor rígido (tipo 2), e $h_{2} / h$, no caso dos agentes do setor flexível (tipo 1 ). $\mathrm{O}$ número de empresas que potencialmente podem mudar de um setor para outro, em ambos os sentidos, é igual ao número de agentes em cada população vezes essas probabilidades, ou seja, $h_{2} h_{1} / h$ em ambos os casos.

$\mathrm{Na}$ comparação de resultados, supomos que os colegiados de trabalhadores levem em consideração os salários reais e a probabilidade de encontrar emprego no setor 2 , que consideramos igual à taxa de emprego vigente nesse setor, $n_{2} /\left(1-n_{1}\right)$. Uma forma simples de formalizar essas considerações é supor que os colegiados decidam com base na diferença dos salários esperados, $\omega_{1}-\omega_{2} n_{2} /\left(1-n_{1}\right)$.

Evidentemente, não há por que supor que todos os agentes revisores reajam da mesma forma a essa diferença. Para um mesmo diferencial, alguns podem julgá-lo suficiente para justificar uma mudança de estratégia, outros não. Ademais, num ambiente de racionalidade limitada, é possível que os resultados não sejam perfeitamente observáveis, principalmente no que concerne à taxa de desemprego. Ou seja, pode haver erros de observação por parte dos agentes revisores, de maneira que mesmo com preferências similares estes agentes reajam de formas distintas a uma mesma diferença $\omega_{1}-\omega_{2} n_{2} /\left(1-n_{1}\right)$. Para incorporar esses aspectos, supomos que a mudança de estratégia de um agente revisor do tipo $i$ só se efetive caso a diferença de salários esperados, obtida pela comparação em pares, seja superior a uma diferença

9 Para formas alternativas de dedução de dinâmicas evolucionárias, ver Vega-Redondo (1996). 
mínima $\varepsilon_{i}$, que definimos como sendo uma variável aleatória com função densidade de probabilidade uniforme com suporte $[-a, a]$, onde $a=\max \left\{\left|\omega_{1}-\omega_{2} n_{2}\right|\right.$ $\left./\left(1-n_{1}\right) \mid\right\} \cdot{ }^{10}$

Segue-se que a probabilidade de um agente revisor do tipo $i$, que compara seu salário esperado com o do agente $j$, mudar de estratégia é:

$$
F(z)=\operatorname{Prob}\left(\varepsilon_{i}<z\right)=\int_{-a}^{z} \frac{1}{2 a} d \varepsilon_{i}=\frac{a+z}{2 a}
$$

onde $z$ é a diferença entre o salário esperado da estratégia alternativa e o da que está sendo adotada. Assim, para uma dada diferença de salários esperados, observada corretamente ou não, todos os agentes revisores cujos $\varepsilon^{\prime} s$ são inferiores a essa diferença mudam de estratégia, sendo a probabilidade de isso ocorrer dada por (4.6).

Resumindo, a probabilidade de um agente do tipo $i$ tornar-se um revisor potencial é $h_{j} / h, j \neq i$; a probabilidade de um agente do tipo $i$ que está reavaliando sua estratégia se tornar um agente do tipo $j$ é $F(z)$. Supondo que estes eventos sejam estatisticamente independentes e multiplicando estas probabilidades, obtemos as probabilidades de os agentes mudarem de setor:

$$
\begin{aligned}
& \text { setor } 2 \text { para } 1: \frac{h_{1}}{h}\left(\frac{a+\omega_{1}-\frac{\omega_{2} n_{2}}{1-n_{1}}}{2 a}\right) \\
& \text { setor } 1 \text { para } 2: \frac{h_{2}}{h}\left(\frac{a+\frac{\omega_{2} n_{2}}{1-n_{1}}-\omega_{1}}{2 a}\right)
\end{aligned}
$$

Como as taxas de revisão por unidade de tempo são iguais a um, o influxo e efluxo de firmas do setor flexível são obtidos multiplicando o número de agentes por essas probabilidades condicionais. Temos, então:

$$
\begin{aligned}
& \dot{h}_{1}^{i}=\frac{h_{2} h_{1}}{h}\left(\frac{a+\omega_{1}-\frac{\omega_{2} n_{2}}{1-n_{1}}}{2 a}\right) \\
& \dot{h_{1}^{e}}=\frac{h_{1} h_{2}}{h}\left(\frac{a-\omega_{1}+\frac{\omega_{2} n_{2}}{1-n_{1}}}{2 a}\right)
\end{aligned}
$$

10 Observe que se trata do mesmo parâmetro $a$ da hipótese sobre a função de produção de (3.1). 
Tendo em vista (4.1), o resultado líquido pode ser expresso como

$$
\dot{\alpha}=\frac{\alpha(1-\alpha)}{a}\left(\omega_{1}-\frac{\omega_{2} n_{2}}{1-n_{1}}\right)
$$

Considere, agora, as decisões dos trabalhadores desempregados, as quais, como afirmamos anteriormente, são estritamente individuais. Um trabalhador nessa situação possui duas alternativas: oferece os seus serviços no setor flexível ou aguarda por uma eventual contratação no setor rígido no período seguinte. Para formalizar as iniciativas dos desempregados, supomos que eles selecionem uma empresa aleatoriamente de acordo com uma distribuição uniforme e comparem o salário vigente nessa empresa com o seu salário de reserva. Aceitar um emprego no setor l significa para o trabalhador desempregado uma revisão de estratégia. De fato, ou ele já era um desempregado e havia optado por tentar novamente um emprego no setor rígido, ou estava empregado nesse setor e perdeu o emprego. Por tratar-se de um estrategista do setor rígido, é plausível assumir que o seu salário reserva guarde alguma relação com o salário do setor no qual ele estava empregado. Supomos, por simplicidade e conveniência matemática, que o seu salário reserva seja igual ao salário vigente nesse setor ponderado pela taxa de desemprego. Assim, se a comparação se der com uma empresa do setor rígido, ele não revê sua estratégia; se a comparação for com uma empresa do setor flexível, a probabilidade condicional de migração é dada por (4.7). Logo, para uma população de tamanho $U$, o efluxo do contingente de desempregados para o setor 1 , normalizado pelo total de trabalhadores da economia, é:

$$
\frac{\dot{N}_{1}^{i 2}}{N}=\frac{U}{N} \alpha\left(\frac{a+\omega_{1}-\frac{\omega_{2} n_{2}}{1-n_{1}}}{2 a}\right)=\frac{\alpha\left(1-n_{1}-n_{2}\right)}{2 a}\left(a+\omega_{1}-\frac{\omega_{2} n_{2}}{1-n_{1}}\right)
$$

Observe que se coexistem os dois setores na economia e há desempregados, esse efluxo será sempre positivo. Ademais, ceteris paribus, a probabilidade de um mesmo trabalhador continuar desempregado diminui ao longo do tempo (e tende a zero quando $t$ tende a infinito).

O fluxo total de trabalhadores para o setor flexível é obtido somando-se os dois influxos e subtraindo o efluxo, ou seja, substituindo-se (4.12), (4.19) e (4.10) em (4.5):

$$
\dot{n}_{1}=\frac{\alpha-n_{1}}{2}+\frac{n_{1}+\alpha-2 n_{1} \alpha}{2 a}\left(\omega_{1}-\frac{\omega_{2} n_{2}}{1-n_{1}}\right)
$$




\section{O MÉDIO PRAZO}

\subsection{O Espaço de Estados e a Fronteira de Pleno Emprego}

Considere o sistema dinâmico formado pela equações (4.11) e (4.13) apresentadas na seção precedente. Substituindo o salário e o nível de emprego pelos valores de equilíbrio de curto prazo (3.22) e (3.26), obtemos o sistema dinâmico do médio prazo:

$$
\begin{aligned}
& \dot{\alpha}=\frac{\alpha(1-\alpha)}{a} \Psi\left(\alpha, n_{1}, A\right) \\
& \dot{n}_{1}=\frac{\alpha-n_{1}}{2}+\frac{n_{1}+\alpha-2 n_{1} \alpha}{2 a} \Psi\left(\alpha, n_{1}, A\right) \equiv \Gamma\left(\alpha, n_{1}, A\right)
\end{aligned}
$$

onde

$$
\Psi\left(\alpha, n_{1}, A\right) \equiv f^{\prime}\left(\frac{c n_{1}}{\alpha}\right)-\frac{\phi\left(\alpha, n_{1}, A\right) n_{2}^{*}}{1-n_{1}}
$$

O espaço de fases é definido a partir do conjunto $\Omega_{1}$ e da fronteira de pleno emprego, $n_{1}+n_{2}^{*}=1$, como segue: ${ }^{11}$

$$
\Omega=\left\{\left(\alpha, n_{1}\right) \in R^{2}: 0<\alpha<1,0<n_{1}<1, n_{1}+n_{2}^{*} \leq 1\right\} \cup\{(0,0),(1,1)\}
$$

A localização da fronteira do pleno emprego e suas alterações em decorrência de alterações de $A$ são necessárias para compreendermos o espaço de fases do modelo. Para tanto, podemos identificar as propriedades apresentadas na proposição a seguir:

Proposição 5.1. (1) A fronteira de pleno emprego, $n_{1}+n_{2}^{*}=1$, sempre passa pelo ponto $(1,1)$ do espaço de fases; (2) o intercepto da fronteira com o eixo $n_{1}$ de $R_{+}^{2} \supset \Omega$ depende negativamente do parâmetro $A ; e$, (3) para $\left(\alpha, n_{1}\right) \in$ int $\Omega$, a fronteira do pleno emprego pode coincidir com o eixo de 45 graus do espaço de fases, mas não pode interceptá-lo.

11 À primeira vista, o sistema apresenta indeterminações nos vetores nulo e unitário, mas elas podem ser contornadas com o uso de limites, como mostraremos posteriormente ao discutirmos os equilíbrios do sistema dinâmico. 
Prova: (1) Substituindo $n_{1}=1$ na definição da fronteira, obtemos $1+n_{2}^{*}=1$. Considerando (3.26), se $\alpha=1$, então $n_{2}^{*}=0$. Logo, a equação da fronteira é satisfeita no equilíbrio clássico.

(2) Considere o conjunto $\Omega_{1}$ definido em (3.3). Primeiro, observe que se $\alpha$ é nulo, a fronteira é $n_{1}+g\left(\phi\left(0, n_{1}, A\right)\right) / c=1$. Como $\phi\left(0, n_{1}, A\right)$ é função constante em relação a $n_{1}$, há solução $0 \leq n_{1} \leq 1$ sempre que $0 \leq g / c \leq 1$. Essas desigualdades podem ser atendidas, pois $g \in[0, \infty)$. Ou seja, a depender dos parâmetros $c$ e $A$, a fronteira pode interceptar o eixo $n_{1}$. Segundo:

$$
\frac{d n_{1}}{d A}=-\frac{g^{\prime} \phi_{A}}{c}<0, \quad n_{1} \geq 0
$$

Assim, elevações de $A$ deslocam esse intercepto para baixo, permanecendo fixo o ponto $(1,1) \cdot{ }^{12}$

(3) Considere o seguinte sistema:

$$
\begin{aligned}
& \alpha=n_{1} \\
& n_{1}+n_{2}=1 \text { : } \\
& D\left(f^{\prime}\left(\frac{c n_{2}}{1-\alpha}\right), A\right)-\alpha \quad f\left(\frac{c n_{1}}{\alpha}\right)-(1-\alpha) f\left(\frac{c n_{2}}{1-\alpha}\right)=0
\end{aligned}
$$

A solução para $\alpha, n_{1}$ e $n_{2}$ identifica os pontos que, simultaneamente, pertencem ao eixo de 45 graus, à fronteira de pleno emprego e solucionam o modelo de curto prazo. O nosso propósito é mostrar que se existe solução, então todo $\alpha=n_{1}$, tal que $\left(\alpha, n_{1}\right) \in$ int $\Omega_{1}$, também resolve o sistema. Substituindo as duas primeiras equações na última, obtemos $D\left(f^{\prime}(c), A\right)-f(c)=0$. A equação envolve apenas parâmetros. Como a derivada do lado esquerdo dessa equação em relação a $A$ é $D_{2}>0$, se existe um $A$ que elimina o excesso de demanda, ele é único. Admita que esse seja o valor de $A$. O sistema fica reduzido às duas primeiras equações que

12 Todavia, se $g / c$ for maior do que um, a fronteira não intercepta o eixo das ordenadas para $n_{1} \geq 0$, o que implica que o vetor $(0,0)$ deixa de pertencer ao espaço de fases. Esse resultado é condizente com a análise do equilíbrio keynesiano puro de curto prazo. Com efeito, havíamos demonstrado que para valores altos de $A$ a solução de curto prazo (que atendesse à restrição de pleno emprego) poderia não existir. 
compreendem três variáveis. Logo, temos um grau de liberdade e o sistema é resolvido para quaisquer $\alpha=n_{1}$. Por conseguinte, para esse específico $A$, a fronteira de pleno emprego coincide com o eixo de 45 graus.

\subsection{Os Equilibrios do Médio Prazo}

No médio prazo, o modelo comporta três tipos de equilíbrio: keynesiano, clássico e de estratégia mista. Como as equações do sistema dinâmico não são definidas nos vetores nulo e unitário, que correspondem às situações keynesiana e clássica, podemos redefinir o sistema dinâmico assumindo que os valores das expressões do lado direito de (5.1) e (5.2) são iguais aos seus limites nesses casos. Para caracterizá-los como equilíbrios basta, então, assegurar que esses limites são iguais a zero quando $\left(\alpha, n_{1}\right)$ tende a $(0,0)$ e $(1,1)$ para qualquer seqüência pertencente ao espaço de fases.

Equilíbrio keynesiano: $\left(\alpha, n_{1}\right)=(0,0)$. Considere a primeira equação do sistema dinâmico, (5.1). Evidentemente, $f^{\prime}\left(\frac{c n_{1}}{\alpha}\right)$ não é definida no vetor nulo. No entanto, as hipóteses sobre a função de produção asseguram que quaisquer que sejam as seqüências $x_{i}^{s}=\left(\alpha^{s}, n_{1}^{s}\right) \in \Omega$, tais que $\lim _{s \rightarrow \infty} x_{1}^{s}=(0,0)$, existe uma vizinhança em torno de $(0,0)$ na qual $f^{\prime}(\cdot) \in[0, a]$, ou seja, a função é limitada quando $\left(\alpha, n_{1}\right)$ tende a $(0,0)$. Ademais, $\phi(\cdot)$ e $n_{2}^{*}=(1-\alpha) g(\phi(\cdot)) / c$ são definidas e contínuas no vetor nulo. Segue-se, então, que

$$
\lim _{\left(\alpha, n_{1}\right) \rightarrow(0,0)} \frac{\alpha(1-\alpha)}{a}\left(f^{\prime}\left(\frac{c n_{1}}{\alpha}\right)-\frac{\phi(\cdot) n_{2}^{*}}{\left(1-n_{1}\right)}\right)=0
$$

O mesmo raciocínio pode ser aplicado à segunda equação do sistema, (5.2), visto que $\left(\alpha-n_{1}\right) / 2$ e o coeficiente $\left(n_{1}+\alpha-2 n_{1} \alpha\right) / 2 a$, que multiplica diferença de salários, tendem a zero quando $\left(\alpha, n_{1}\right) \rightarrow(0,0)$.

Equilíbrio clássico: $\left(\alpha, n_{1}\right)=(1,1)$. No que tange a (5.1), o modelo comporta o equilíbrio clássico somente se o seguinte limite for igual a zero:

$$
\lim _{\left(\alpha, n_{1}\right) \rightarrow(1,1)} \frac{\alpha(1-\alpha)}{a} f^{\prime}\left(\frac{c n_{1}}{\alpha}\right)+\lim _{\left(\alpha, n_{1}\right) \rightarrow(1,1)}-\frac{\alpha(1-\alpha)}{a} \frac{\phi(\cdot) n_{2}^{*}}{\left(1-n_{1}\right)}
$$


O primeiro limite é, obviamente, igual a zero. Em relação ao segundo, observe que, como estamos interessados apenas nos limites para seqüências pertencentes a $\Omega$, vale a restrição da fronteira de pleno emprego, o que implica $n_{2}^{*} \leq 1-n_{1}$. Logo, para qualquer $n_{1}$ tal que $0 \leq n_{1}<1$, temos

$$
\begin{aligned}
& \max \left\{\frac{\phi(\cdot) n_{2}^{*}}{1-n_{1}}\right\}=\max \{\phi(\cdot)\} \times \max \left\{\frac{n_{2}^{*}}{1-n_{1}}\right\}= \\
& =a \times \max \left\{\frac{1-n_{1}}{1-n_{1}}\right\}=a ; \mathrm{e} \\
& \min \left\{\frac{\phi(\cdot) n_{2}^{*}}{1-n_{1}}\right\}=0 .
\end{aligned}
$$

Logo, $\phi(\cdot) n_{2}^{*} /\left(1-n_{1}\right)$ é limitada e, portanto, o limite do segundo termo de (5.10) também é igual a zero. Demonstração análoga vale para a segunda equação do sistema dinâmico, pois se $\left(\alpha, n_{1}\right)$ tende $(1,1)$, tanto $\left(\alpha-n_{1}\right) / 2$ quanto $\left(n_{1}+\alpha-2 n_{1} \alpha\right) / 2 a$ tendem a zero.

Equilibrios de estratégia mista. As situações clássica e keynesiana puras não esgotam as possibilidades de equilíbrio. Considere a equação do fluxo de firmas. Qualquer $\left(\alpha, n_{1}\right)$ tal que a diferença de salários é zero anula $\dot{\alpha}$. Já no que concerne ao fluxo de trabalhadores, se $\alpha$ for igual a $n_{1}$ e a diferença de salários nula, então $\dot{n}_{1}$ é igual a zero. Logo, existe um terceiro conjunto de equilíbrios que é formado pelos vetores que atendem a essas condiçôes, ou seja:

$$
\forall\left(\alpha, n_{1}\right): 0<\alpha=n_{1}<1, f^{\prime}\left(\frac{c n_{1}}{\alpha}\right)-\frac{\phi(\cdot) n_{2}^{*}}{1-n_{1}}=0
$$

Todos os equilíbrios situam-se, portanto, no eixo de 45 graus. Os de estratégia mista são, normalmente, equilíbrios com desemprego, pois a fronteira de pleno emprego não tem pontos em comum com o eixo de 45 graus, a não ser no caso de ela coincidir com esse eixo, situação na qual temos infinitos equilíbrios, mas que só ocorre para um único valor de $A$ (cf. proposição 5.1). 


\subsection{Análise dos equilibrios de estratégia mista}

Existem equilíbrios de estratégia mista somente se a diferença de salários é nula, condição que, tendo em vista (3.26), pode ser expressa como:

$$
f^{\prime}\left(\frac{c \quad n_{1}}{\alpha}\right)-\frac{\omega_{2}^{*}(1-\alpha) g\left(\omega_{2}^{*}\right)}{c\left(1-n_{1}\right)}=0
$$

Como $n_{1}$ deve ser igual a $\alpha$ nesses equilíbrios, obtemos:

$$
f^{\prime}(c) c=\omega_{2}^{*} g\left(\omega_{2}^{*}\right)
$$

Existem, portanto, tantos equilíbrios de estratégia mista quantas forem as soluções dessa equação para $\alpha=n_{1} \in(0,1)$.

Considere, inicialmente, a solução $\omega_{2}^{*}=f^{\prime}(c)$, representada na Figura 2 pelo ponto $Q$. A equação $(5.15)$ reduz a $c=g\left(\omega_{2}^{*}\right)$. Substituindo $g\left(\omega_{2}^{*}\right)$ por $c n_{2}^{*} /(1-\alpha)$ e, em seguida, $\alpha$ por $n_{1}$, obtemos $n_{1}+n_{2}^{*}=1$. Vigora, portanto, o pleno emprego e há infinitos equilíbrios, pois, como demonstrado na proposição 5.1, no caso $\alpha=n_{1}$ a fronteira de pleno emprego é o próprio eixo de 45 graus. Todavia, essa situação depende de um específico valor para o parâmetro $A$, motivo pelo qual escusamo-nos de analisar esse caso. O mesmo raciocínio permite-nos descartar, como candidato a equilíbrio de estratégia mista, qualquer $\alpha$ tal que $g\left(\omega_{2}^{*}\right)>c$, pois isso implica $n_{2}^{*}+n_{1}>1$. 
FIGURA 2 - FUNÇÃO INVERSA DE PRODUTIVIDADE MARGINAL DAS FIRMAS DO TIPO 2

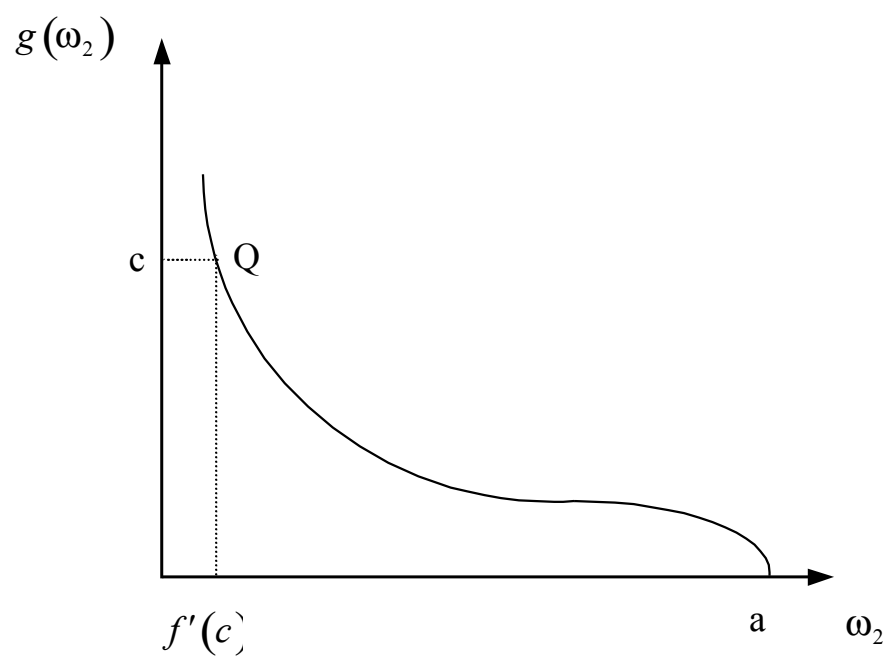

Voltemo-nos, então, para o exame de equilíbrios de estratégia mista com desemprego. Identificar equilíbrios desse tipo significa examinar se existem $n_{1}=\alpha$ tais que os salários reais do setor 2 geram massas de salários $\omega_{2} g\left(\omega_{2}\right)$ iguais a $f^{\prime}(c) c$. Podemos, portanto, discutir esses equilíbrios por meio do exame do comportamento da função $\omega_{2} g\left(\omega_{2}\right)$ para valores $\omega_{2}$ gerados por estados em que $n_{1}=\alpha$.

Devido às hipóteses a respeito da função de produção (3.1), se o salário assume seu valor máximo, $a$, então $g\left(\omega_{2}\right)$ é igual a zero. Logo, para $\omega_{2}=a$, a função $\omega_{2} g\left(\omega_{2}\right)$ é nula. Além disso, $\omega_{2} g\left(\omega_{2}\right)$ é contínua e, como vimos acima, igual a $c f^{\prime}(c)$ quando $\omega_{2}$ é igual a $f^{\prime}(c)$. Dois formatos possíveis de $\omega_{2} g\left(\omega_{2}\right)$ são apresentados na Figura 3. 


\section{FIGURA 3 - FUNÇÃO MASSA DE SALÁRIO DAS FIRMAS TIPO 2}

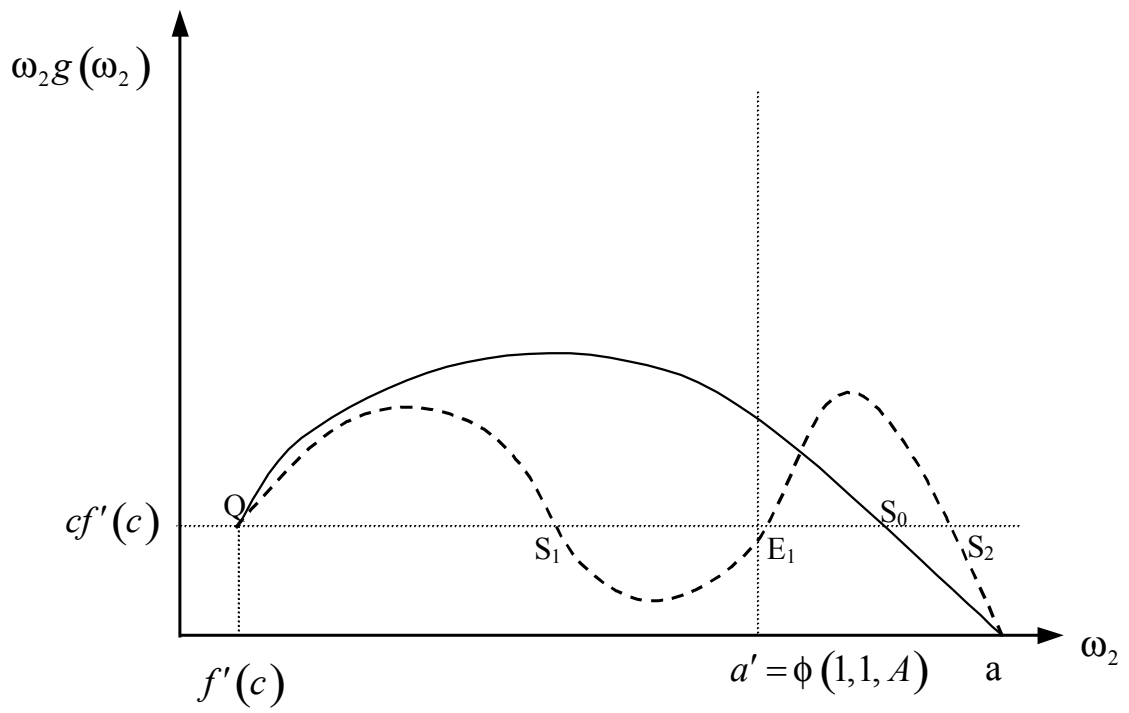

Nessa figura, a curva cheia intercepta, por construção, a reta $c f^{\prime}(c)$ nos pontos $Q$ e $S_{0}$. Temos, então, um único equilíbrio de estratégia mista. Já no caso da curva tracejada, há três equilíbrios, representados pelos pontos $S_{1}, E_{1}$ e $S_{2}$.

Assumimos, até agora, que $\max \left\{\omega_{2}^{*}\right\}=a$. No entanto, no sistema dinâmico $\omega_{2}^{*}$ pode não atingir esse máximo. Com efeito, observe que existe uma relação perfeitamente determinada entre $\omega_{2}^{*}$ e $\alpha$ ao longo do eixo de 45 graus, ou seja, a derivada total é:

$$
\left.\frac{d \omega_{2}^{*}}{d \alpha}\right|_{\substack{d A=0, \alpha=n_{1}, d \alpha=d n_{1}}}=\phi_{\alpha}+\phi_{n_{1}}>0
$$

De fato, recordando (3.29) e (3.27), temos:

$$
\phi_{\alpha}+\phi_{n_{1}}=\frac{f\left(\frac{c n_{1}}{\alpha}\right)-\frac{c n_{1}}{\alpha} f^{\prime}\left(\frac{c n_{1}}{\alpha}\right)-f\left(g\left(\omega_{2}\right)\right)+c f^{\prime}\left(\frac{c n_{1}}{\alpha}\right)}{D_{1}-(1-\alpha) \omega_{2} g^{\prime}\left(\omega_{2}\right)}
$$

Como $g\left(\omega_{2}\right)=c n_{2} /(1-\alpha)$ e essas derivadas são calculadas em $\alpha=n_{1}$, temos que: 


$$
\phi_{\alpha}+\phi_{n_{1}}=\frac{f(c)-f\left(\frac{c n_{2}}{1-n_{1}}\right)}{D_{1}-(1-\alpha) \omega_{2} g^{\prime}\left(\omega_{2}\right)}>0
$$

pois, sob a hipótese de desemprego, $n_{2}<1-n_{1}$.

Logo, quando consideramos os pontos pertencentes ao eixo de 45 graus, o valor máximo que $\omega_{2}^{*}$ pode assumir é $\phi(1,1, A) \equiv a^{\prime}$. Como sabemos que $\phi_{A}$ é negativo, quanto maior for $A$, menor será esse salário máximo $a^{\prime}$. Segue-se, portanto, que o domínio da função $\omega_{2} g\left(\omega_{2}\right)$ é o intervalo $\left[f^{\prime}(c), a^{\prime}\right]$, no qual o extremo inferior é fixo e o superior depende inversamente de $A$, sendo $a^{\prime} \leq a$. Para um $A$ suficientemente grande, o domínio reduz-se à singularidade $f^{\prime}(c)$, situação que, como vimos, corresponde ao caso de infinitos equilíbrios de estratégia mista de pleno emprego. Evidentemente, variações de $A$ e, por conseguinte, de $a^{\prime}$, alteram o número de equilíbrios de estratégia mista com desemprego para uma mesma função $\omega_{2} g\left(\omega_{2}\right)$.

O modelo comporta, portanto, uma multiplicidade de configurações estruturais a depender dos valores dos parâmetros $c$ e $A$, que determinam o domínio $\left[f^{\prime}(c), a^{\prime}\right]$ e o formato da função $\omega_{2} g\left(\omega_{2}\right)$. Considere, por exemplo, a curva pontilhada, com situação inicial $a^{\prime}=a$, e admita aumentos contínuos dos gastos do governo. Inicialmente, temos a configuração $S_{1}-E_{1}-S_{2}$. À medida que $A$ aumenta, a economia apresenta, sucessivamente, as configurações: (i) $S_{1}-E_{1}$; (ii) $S_{1}$; (iii) inexistência de equilíbrios de estratégia mista com desemprego; (iv) infinitos equilíbrios de pleno emprego.

É importante notar que, para um dado valor do parâmetro $A$, os equilíbrios de estratégia mista mais próximos do equilíbrio clássico apresentam menores taxas de desemprego. Com efeito, considere a taxa de desemprego em função do estado do sistema:

$$
u=1-n_{1}-\frac{1-\alpha}{c} g\left(\phi\left(\alpha, n_{1}, A\right)\right)
$$

Ao calcularmos o diferencial total dessa função para valores ao longo da reta de 45 graus, $n_{1}=\alpha$ e $d n_{1}=d \alpha$, e considerando $d A=0$, obtemos: 


$$
\left.\frac{d u}{d \alpha}\right|_{\substack{d A=0, n_{1}=\alpha, d n_{1}=d \alpha}}=\frac{g-c-(1-\alpha) g^{\prime}\left(\phi_{\alpha}+\phi_{n_{1}}\right)}{c}
$$

Usando (3.27) e (3.28), é possível reescrever essa derivada:

$$
\left.\frac{d u}{d \alpha}\right|_{\substack{d A=0, n_{1}=\alpha, d n_{1}=d \alpha}}=\frac{(1-\alpha)(f(c)-f(g)) g^{\prime}+(c-g)\left(D_{1}-(1-\alpha) \phi g^{\prime}\right)}{c(1-\alpha)\left(D_{1}-(1-\alpha) \phi g^{\prime}\right) g^{\prime}}
$$

Observe que o denominador desta derivada é indubitavelmente negativo. O sinal desta derivada depende, então, do sinal do numerador, que passamos a analisar. Por simples manipulação algébrica, podemos reescrever o numerador em questão como

$$
(c-g) D_{1}+(1-\alpha)\{f(c)-f(g)-(c-g) \phi\} g^{\prime}
$$

Nos estados de equilíbrio de estratégia mista com desemprego, temos $n_{2}<1-n_{1}=1-\alpha$. Logo, $c>\frac{c n_{2}}{1-\alpha}=g$, ou seja, $c-g>0$. Como a função de produção é, por hipótese, estritamente côncava, $\frac{f(c)-f(g)}{c-g}<f^{\prime}(g)=\phi$. Logo, a expressão entre chaves em (5.22) apresenta sinal negativo, o que é uma condição suficiente para que (5.22) como um todo tenha sinal positivo. Isto demonstra que a derivada (5.2l) é negativa, ou seja, as taxas de desemprego são menores em equilíbrios de estratégia mista com setor clássico relativamente maior.

Os equilíbrios $S$ e $E$ distinguem-se pelo sinal da derivada de $\omega_{2} g\left(\omega_{2}\right)$ : no primeiro caso, esse sinal é negativo; no segundo, positivo. O cálculo dessa derivada resulta na seguinte expressão:

$$
\frac{\partial\left(\omega_{2} g\left(\omega_{2}\right)\right)}{\partial \omega_{2}}=g\left(\omega_{2}\right)+\omega_{2} g^{\prime}\left(\omega_{2}\right)
$$

Então, esse sinal está associado à elasticidade da função inversa da produtividade marginal: se o sinal for positivo, a elasticidade é menor do que zero e maior do que -1 ; se for negativo, a elasticidade é menor do que -1 . Ou seja, em última instância, a existência e o número de equilíbrios de estratégia mista dependem de hipóteses a respeito da terceira derivada da função de produção. A elasticidade determina, igualmente, as propriedades dinâmicas desses equilíbrios. Como demonstraremos a seguir, os equilíbrios $S$ são selas e os $E$ assintoticamente estáveis. 
A matriz jacobiana da linearização em torno dos equilíbrios de estratégia mista é

$$
\left.J\right|_{\alpha=n_{1}, \Psi=0}=\left[\begin{array}{cc}
\frac{\alpha(1-\alpha) \Delta_{\alpha}}{a} & \frac{\alpha(1-\alpha) \Delta_{n_{1}}}{a} \\
\frac{1}{2}+\frac{\alpha(1-\alpha) \Delta_{\alpha}}{a} & -\frac{1}{2}+\frac{\alpha(1-\alpha) \Delta_{n_{1}}}{a}
\end{array}\right]
$$

onde $\Delta_{i}=\frac{\omega_{2} g\left(\omega_{2}\right)}{c(1-\alpha)}-\frac{\phi_{i}\left(g\left(\omega_{2}\right)+\omega_{2} g^{\prime}\left(\omega_{2}\right)\right)}{c}-\frac{c f^{\prime \prime}(c)}{\alpha}, i=\alpha, n_{1}$, cujos autovalores são:

$$
\left\{-\frac{1}{2},-\frac{\alpha(1-\alpha)\left(\phi_{\alpha}+\phi_{n_{1}}\right)\left(g\left(\omega_{2}\right)+\omega_{2} g^{\prime}\left(\omega_{2}\right)\right)}{c a}\right\}
$$

Dado que nos equilíbrios de estratégia mista $\phi_{\alpha}+\phi_{n_{1}}>0$, o sinal do segundo autovalor é determinado inequivocamente pelo sinal de $g\left(\omega_{2}\right)+\omega_{2} g^{\prime}\left(\omega_{2}\right)$. Se esse sinal for positivo, os dois autovalores são negativos e o equilíbrio é assintoticamente estável; se for negativo, os autovalores apresentam sinal contrário e temos instabilidade de sela.

Em síntese, as hipóteses que fizemos ao longo do texto são suficientes para permitir a existência de equilíbrios de estratégia mista. Eles podem ser múltiplos, assintoticamente estáveis ou selas. A presença de um equilíbrio estável significa que há uma bacia de atração, tal que se o equilíbrio de curto prazo situar-se nessa região, a economia converge para um equilíbrio de médio prazo com desemprego. Ademais, os equilíbrios de estratégia mista são, necessariamente, equilíbrios de fluxos e não de estoques. Com efeito, recuperando as equações de influxo e efluxo, (4.3) e (4.4), constatamos que $\dot{h}_{1}^{i}=\dot{h}_{1}^{e}=h_{2} h_{1} / 2 h>0$. Ou seja, ambos são positivos e sua diferença se anula. Por conseguinte, mesmo nesses equilíbrios existem fluxos entre as três subpopulações de trabalhadores. Em particular, trabalhadores empregados perdem suas ocupações e trabalhadores desempregados obtêm ocupaçóes tanto no setor keynesiano quanto no setor clássico. Em outros termos, a economia convive com uma determinada taxa de desemprego, não existem forças endógenas que eliminem esse desemprego mesmo no médio prazo, mas não são sempre os mesmos trabalhadores que permanecem sem emprego.

Por fim, observe o papel crucial desempenhado pelo parâmetro $A$. Não só ele altera a fronteira de pleno emprego, e, portanto, o espaço de fases do modelo, como também o número de equilíbrios para uma mesma função de produção. A política econômica adquire, por extensão, uma relevância para determinação do nível de empre- 
go de médio prazo que lhe foi, de certo modo, negada na análise tradicional do modelo keynesiano.

\subsection{Análise dos Equilibrios de Estratégia Pura}

$\mathrm{Na}$ discussão das propriedades dos equilíbrios clássico e keynesiano, vamos nos restringir ao caso mais simples no qual existe um único equilíbrio de estratégia mista de sela, que designaremos como $\left(\alpha^{*}, n_{1}^{*}\right)$. Como o sistema dinâmico não é continuamente diferenciável nos dois equilíbrios de estratégia pura, é impossível aplicar a técnica tradicional de linearização. Devemos, então, proceder a uma análise qualitativa.

Considere a curva de demarcação $\Psi\left(\alpha, n_{1}\right)=0$, associada a (5.1). ${ }^{13}$ A seu respeito valem as seguintes proposições que permitem identificar sua localização no espaço de fases.

Proposição 5.2. Existe uma função contínua $\psi(\alpha) \in(0,1)$, tal que $\Psi(\alpha, \psi(\alpha))=0$ para todo $\alpha \in(0,1)$.

Prova: Existência da função: se $n_{1}=0$, a diferença de salários é:

$$
\Psi(\alpha, 0)=a-\phi(\alpha, 0) n_{2}^{*}
$$

Sabemos que $n_{2}^{*} \in[0,1]$ e $\phi \in(0, a]$. Ademais, o nível de emprego e o salário do setor 2 são inversamente relacionados, em particular, se o salário do setor 2 for máximo, então $n_{2}^{*}=0$. Logo, $\phi n_{2}^{*}<a$ e, portanto, $\Psi(\alpha, 0)>0$.

Para $n_{1}=1$, a função $\Psi(\cdot)$ não está definida. No entanto, temos que:

$$
\lim _{n_{1} \rightarrow 1^{-}} \Psi\left(\alpha, n_{1}\right)=f^{\prime}\left(\frac{c}{\alpha}\right)-\lim _{n_{1} \rightarrow 1^{-}} \frac{\phi(\cdot)(1-\alpha) g(\phi(\cdot))}{c\left(1-n_{1}\right)}
$$

Como a massa salarial de uma firma do setor $2, \phi(\cdot) g(\phi(\cdot))$, é menor do que o produto máximo total, $b$, ela é limitada. Assim, para qualquer $\alpha \in(0,1)$,

13 Como a análise restringe-se ao caso de equilíbrio único, o valor de $A$ está, por definição, fixo. Por economia de notação omitimos essa variável nas funções $\Psi$ e $\Gamma$. 


$$
\lim _{n_{1} \rightarrow 1^{-}} \Psi\left(\alpha, n_{1}\right)=f^{\prime}\left(\frac{c}{\alpha}\right)-\infty=-\infty
$$

Como $\Psi(\cdot)$ é contínua, para todo $\alpha \in(0,1)$ existe, pelo TVI, pelo menos um $n_{1} \in(0,1)$ que anula a diferença de salários esperados. Garantida a existência de solução, provemos a unicidade.

Considere a seguinte derivada:

$$
\Psi_{n_{1}}=-\frac{\omega_{2}^{*}(1-\alpha) g\left(\omega_{2}^{*}\right)}{c\left(1-n_{1}\right)^{2}}-\frac{(1-\alpha) \phi_{n_{1}}\left(g\left(\omega_{2}^{*}\right)+\omega_{2}^{*} g^{\prime}\left(\omega_{2}^{*}\right)\right)}{c\left(1-n_{1}\right)}+\frac{c f^{\prime \prime}\left(\frac{c n_{1}}{\alpha}\right)}{\alpha}
$$

Para avaliarmos essa derivada nos pontos em que a diferença de salários esperados é nula, igualamos (5.3) a zero, obtendo

$$
g\left(\omega_{2}^{*}\right)=\frac{c\left(1-n_{1}\right) f^{\prime}\left(\frac{c n_{1}}{\alpha}\right)}{(1-\alpha) \omega_{2}^{*}}
$$

Ao substituirmos essa equação em (5.29) e utilizarmos (3.29) para eliminar $\phi_{n_{1}}$, temos, após algumas simplificações,

$$
\left.\Psi_{n_{1}}\right|_{\Psi=0}=-\frac{c \alpha f^{\prime}\left(\frac{c n_{1}}{\alpha}\right)\left(D_{1} \omega_{2}^{*}+c\left(1-n_{1}\right) \omega_{2}^{*} f^{\prime}\left(\frac{c n_{1}}{\alpha}\right)\right)}{c\left(1-n_{1}\right) \omega_{2}^{*} \alpha\left(D_{1}-(1-\alpha) \omega_{2}^{*} g^{\prime}\left(\omega_{2}^{*}\right)\right)}+\frac{c f^{\prime \prime}\left(\frac{c n_{1}}{\alpha}\right)}{\alpha}<0 .
$$

pois os dois termos são estritamente negativos para quaisquer $\alpha$ e $n_{1}$ pertencentes ao intervalo $(0,1)$. Assim, a função corta o eixo $n_{1}$ sempre com inclinação negativa. Logo, para cada $\alpha$ há uma única solução $n_{1}$. Ou seja, existe uma função $\psi(\alpha) \in(0,1), \alpha \in(0,1)$, tal que $\Psi(\alpha, \psi(\alpha))=0$.

Continuidade da função: observe que

$$
\lim _{\alpha \rightarrow 0^{+}} \Psi\left(\alpha, n_{1}\right)=-\frac{\phi\left(0, n_{1}\right) g\left(\phi\left(0, n_{1}\right)\right)}{c\left(1-n_{1}\right)}<0 ; \text { e } \Psi\left(1, n_{1}\right)=f^{\prime}\left(c n_{1}\right)>0 .
$$

Segue-se que, para cada $n_{1} \in(0,1)$, existe pelo menos um $\alpha \in(0,1)$, tal que $\Psi(\cdot)=0$. 
Dados esses resultados, a posição de $\Psi(\cdot)=0$ no plano de fases pode ser identificada por meio do exame dos limites de $\psi(\alpha)$, quando $\alpha$ tende a zero e a um, e do sinal de $\partial \psi / \partial \alpha$ no equilíbrio de estratégia mista, i. e., quando essa curva de demarcação corta o eixo de 45 graus.

Proposição 5.3. Se o equilibrio de estratégia mista for uma sela, então, $\frac{d \psi\left(\alpha^{*}\right)}{d \alpha}>1$.

Prova: Se a curva corta o eixo de 45 graus por baixo, devemos ter

$$
\frac{d \psi\left(\alpha^{*}\right)}{d \alpha}=-\left.\frac{\Psi_{\alpha}}{\Psi_{n_{1}}}\right|_{\Psi\left(\alpha^{*}, n_{1}^{*}\right)=0}=\frac{-\frac{\omega_{2}^{*} g(\cdot)}{c(1-\alpha)}+\frac{\phi_{\alpha}\left(g(\cdot)+\omega_{2}^{*} g^{\prime} \cdot(\cdot)\right)}{c}+\frac{c f^{\prime \prime}(c)}{\alpha}}{-\frac{\omega_{2}^{*} g(\cdot)}{c(1-\alpha)}-\frac{\phi_{n_{1}}\left(g(\cdot)+\omega_{2}^{*} g^{\prime}(\cdot)\right)}{c}+\frac{c f^{\prime \prime}(c)}{\alpha}}>1
$$

De (5.31) concluímos que o denominador é negativo, pois $\left.\Psi_{n_{1}}\right|_{\Psi=0}<0 \Rightarrow$ $\left.\Rightarrow \Psi_{n_{1}}\right|_{\left(\alpha^{*}, n_{1}^{*}\right)}<0$. Assim, a desigualdade é atendida se, e somente se,

$$
\left(\phi_{\alpha}+\phi_{n_{1}}\right)\left(g(\cdot)+\omega_{2}^{*} g^{\prime}(\cdot)\right)<0 \text {. }
$$

Sabemos que $\phi_{\alpha}+\phi_{n_{1}}>0$ quando essas derivadas são calculadas em $\alpha=n_{1}$. Logo, a condição (5.34) depende do sinal de $g(\cdot)+\omega_{2}^{*} g^{\prime}(\cdot)$, o qual, como sabemos, determina também as propriedades de estabilidade do equilíbrio de estratégia mista. Assim, a curva de demarcação corta o eixo de 45 graus por baixo se, e somente se, $g(\cdot)+\omega_{2}^{*} g^{\prime}(\cdot)$ for menor do que zero, ou seja, se o equilíbrio de estratégia mista for uma sela.

Observe, para uso posterior, que a proposição implica:

$$
\Psi_{\alpha}+\Psi_{n_{1}}>0
$$

No que concerne aos limites, note que, sob a hipótese de que o equilíbrio é único e é uma sela, a função $\psi(\alpha)>0$ situa-se abaixo do eixo de 45 graus para $\alpha \in\left(0, \alpha^{*}\right)$ e acima desse eixo se $\alpha \in\left(\alpha^{*}, 1\right)$. Assim, a função está limitada superiormente (inferiormente) pelo eixo de 45 graus à esquerda (direita) do equilíbrio de estratégia mista. Segue-se, então, que $\lim _{\alpha \rightarrow 0} \psi(\alpha)=0 ; \quad \mathrm{e} \lim _{\alpha \rightarrow 1} \psi(\alpha)=1$. Um formato possível de $\Psi(\cdot)=0$ é apresentado na Figura 4. Por último, para obter 
as setas direcionais, observe que, como $\Psi_{n_{1}}$ é menor do que zero nos pontos $(\alpha, \psi(\alpha))$, então a diferença salarial $\Psi$ e, portanto, $\dot{\alpha}$ são positivos (negativos) nos pontos abaixo (acima) da curva de demarcação.

Considere, agora, a curva de demarcação $\Gamma\left(\alpha, n_{1}\right)=0$ associada a (5.2). A proposição a seguir garante a existência da curva de demarcação e assegura que ela, por assim dizer, atravessa o espaço de fases.

Proposição 5.4. (1) Para todo $\alpha \in(0,1)$, existe pelo menos um $n_{1}$ tal que $\Gamma\left(\alpha, n_{1}\right)=0$; (2) para cada $n_{1} \in(0,1)$, existe pelo menos um $\alpha \in(0,1)$ tal que $\Gamma(\cdot)=0$.

Prova: (1) Para $n_{1}$ igual a zero, temos

$$
\Gamma(\alpha, 0)=\frac{\alpha}{2}+\frac{\alpha}{2 a} \Psi(\alpha, 0)>0
$$

pois, como mostramos anteriormente (cf. prova da proposição 5.2), $\Psi(\alpha, 0)>0$. Ademais,

$$
\begin{aligned}
& \lim _{n_{1 \rightarrow 1^{-}}} \Gamma\left(\alpha, n_{1}\right)=\frac{\alpha-1}{2}+\frac{1-\alpha}{2 a} \lim _{n_{1 \rightarrow 1^{-}}} \Psi\left(\alpha, n_{1}\right)= \\
& =\frac{\alpha-1}{2}+\frac{1-\alpha}{2 a}(-\infty)=-\infty,
\end{aligned}
$$

Como $\Gamma\left(\alpha, n_{1}\right)$ é contínua, o TVI garante a primeira parte da proposição.

(2) Dados os resultados (5.32), temos:

$$
\begin{aligned}
& \lim _{\alpha \rightarrow 0^{+}} \Gamma\left(\alpha, n_{1}\right)=-\frac{n_{1}}{2}+\frac{n_{1}}{2 a} \lim _{\alpha \rightarrow 0^{+}} \Psi(\cdot)<0 ; \mathrm{e} \\
& \Gamma\left(1, n_{1}\right)=\frac{1-n_{1}}{2 a}\left(a+f^{\prime}\left(c n_{1}\right)\right)>0,
\end{aligned}
$$

o que prova a segunda parte.

Podemos, em seguida, identificar sua localização no plano de fases. 
Proposição 5.5. Se o equilíbrio de estratégia mista for uma sela, a curva $\Gamma(\cdot)=0$ situa-se no interior das regióes delimitadas pela função $\psi(\alpha)$ e o eixo de 45 graus, para todo $n_{1} \neq \alpha$.

Prova: A prova é feita em quatro etapas. Primeiro, se a curva corta o eixo de 45 graus por baixo, temos:

$$
\left.\frac{\partial n_{1}}{\partial \alpha}\right|_{\Gamma\left(\alpha^{*}, n_{1}^{*}\right)=0}=-\frac{\Gamma_{\alpha}}{\Gamma_{n_{1}}}=\frac{a+2(1-\alpha) \alpha \Psi_{\alpha}}{a-2(1-\alpha) \alpha \Psi_{n_{1}}}>1
$$

Como $\Psi_{n_{1}}\left(\alpha^{*}, n_{1}^{*}\right)<0$, o denominador é positivo. Logo, a condição acima é atendida se, e somente se:

$$
2(1-\alpha) \alpha\left(\Psi_{\alpha}+\Psi_{n_{1}}\right)>0
$$

Tendo em vista que $\Psi_{\alpha}+\Psi_{n_{1}}>0$, como assinalado em (5.35), concluímos, então, que, se o equilíbrio de estratégia mista for uma sela, a curva de demarcação corta o eixo por baixo. Segundo, a função $\psi(\alpha)$ é mais inclinada do que $\Gamma(\cdot)=0$ nesse equilíbrio, pois:

$$
\frac{d \psi\left(\alpha^{*}\right)}{d \alpha}-\left.\frac{\partial n_{1}}{\partial \alpha}\right|_{\left(\alpha^{*}, n_{1}^{*}\right)}=-\frac{a\left(\Psi_{\alpha}+\Psi_{n_{1}}\right)}{\Psi_{n_{1}}\left(a-2(1-\alpha) \alpha \Psi_{n_{1}}\right)}>0
$$

Terceiro, $\Gamma(\cdot)=0$ só intercepta o eixo de 45 graus no equilíbrio de estratégia mista, pois se $n_{1}=\alpha$, temos $\Gamma(\cdot)=\alpha(1-\alpha) \Psi(\cdot) / a$, que só pode ser igual a zero se $\Psi(\cdot)=0$. Quarto, por definição, $\Gamma(\cdot)=0$ só intercepta $\psi(\alpha)$ no equilíbrio de estratégia mista. Conclui-se, então, que para $n_{1} \neq \alpha$, a curva $\Gamma(\cdot)=0$ situa-se no interior das regióes acima identificadas.

Para obter as setas direcionais, considere um ponto $\left(\bar{\alpha}, \bar{n}_{1}\right)$ tal que $\Psi(\cdot)=0$ e $\bar{\alpha}>\alpha^{*}$. Obviamente, $\bar{n}_{1}>\bar{\alpha}$ e $\dot{n}_{1}=\Gamma=\left(\bar{\alpha}-\bar{n}_{1}\right) / 2<0$. Essa direção vale para qualquer estado que possa ser ligado ao ponto $\left(\bar{\alpha}, \bar{n}_{1}\right)$ por um caminho que não intercepte a curva de demarcação $\Gamma(\cdot)=0$. Ou seja, para os pontos de qualquer conjunto $M \subset$ int $\Omega_{1}$, conexo por caminhos, delimitado pela curva de demarcação, 
tal que $\left(\bar{\alpha}, \bar{n}_{1}\right) \in M$ e $n_{1}+n_{2}^{*} \leq 1$. Simetricamente, tomemos um ponto tal que $\Psi(\cdot)=0$ e com abscissa menor do que $\alpha^{*}$. Neste caso, $\dot{n}_{1}>0$ e, portanto, esse sinal vale em qualquer ponto pertencente a um conjunto conexo por caminhos definido de forma similar ao conjunto $M$ acima.

O diagrama de fases, apresentado na figura a seguir, incorpora esses resultados e permite caracterizar os equilíbrios clássico e keynesiano como atratores locais.

FIGURA 4 - DIAGR AMA DE FASES

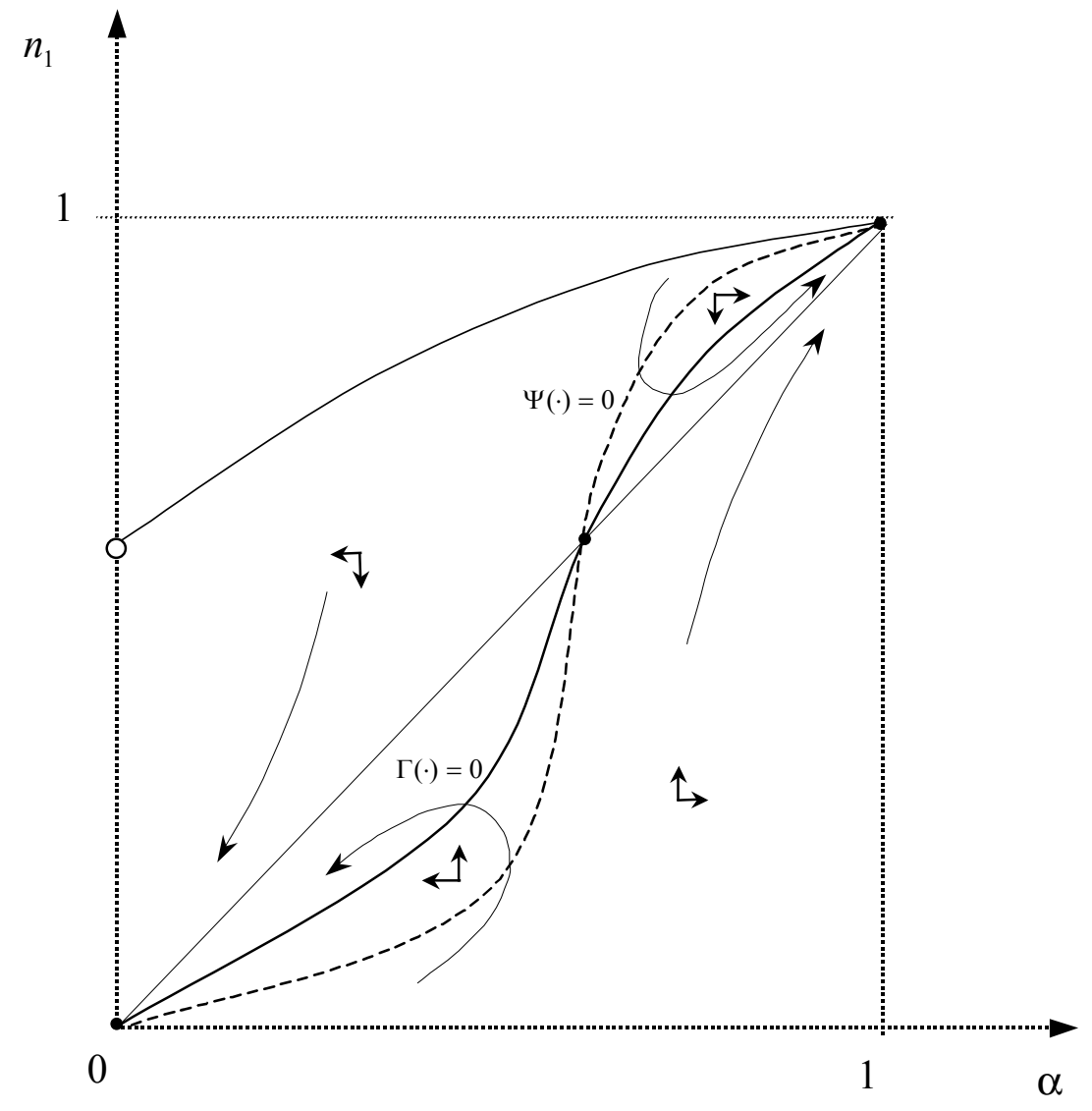

\section{CONCLUSÕES}

A adoção do conceito de equilíbrio evolucionário, em substituição ao tratamento tradicional do mercado de trabalho, permite restaurar a importância da demanda 
agregada na determinação do nível de emprego. O gasto autônomo e as políticas de controle de demanda não têm mais apenas efeitos de curto prazo. Na seção 5 , mostramos que o número de equilíbrios de estratégia mista do médio prazo e as propriedades dinâmicas do sistema dependem crucialmente da demanda. Em particular, o pleno emprego - i. e., o caso clássico ou o de infinitos equilíbrios de estratégia mista - apresenta-se tão-somente como uma das configurações possíveis de uma economia governada pelo princípio da demanda efetiva, como sustentava Keynes. Em outros termos, o pleno emprego não é, no modelo, um estado ao qual tende naturalmente uma economia na qual os agentes perseguem interesses próprios - isto ocorre, é importante observar, mesmo na presença do efeito riqueza. Ele resulta de políticas macroeconômicas adequadas ou de espíritos animais, elementos apreendidos no modelo pelo parâmetro $A$.

O conceito de equilíbrio evolucionário permite, igualmente, um outro olhar para a questão da flexibilidade dos salários. Considere o equilíbrio keynesiano puro da seção 5. Demonstramos que ele é um atrator. Assim, qualquer tentativa de flexibilizar salários nominais por parte de alguns colegiados (uma pequena mutação na linguagem dos jogos evolucionários) apenas desloca a economia para um outro estado da bacia de atração desse equilíbrio. Ou seja, uma flexibilização desse tipo resulta em ganhos inferiores aos da manutenção do salário rígido e tende, portanto, a desaparecer.

Por último, à luz do modelo apresentado, o desemprego não é fruto da mera postulação de uma rigidez nominal. Manter o salário nominal fixo é apenas uma das estratégias possíveis, que só é adotada na medida em que os agentes, pautados em suas percepções dos mercados, a consideram mais proveitosa. Os equilíbrios com desemprego resultam não de uma hipótese $a d$ hoc, mas sim do processo de interação de agentes auto-interessados num ambiente de racionalidade limitada. O desemprego apresenta-se, assim, como propriedade emergente.

\section{REFERENNCIAS}

BLANCHARD, O. Macroeconomia: teoria e política econômica. Rio de Janeiro: Campus, 1999.

BONOMO, M.; CARRASCO, V.; MOREIRA, H. Aprendizado evolucionário, inércia inflacionária e recessão em desinflações monetárias. Revista Brasileira de Economia, v. 57, n. 4, out.-dez. 2003.

DARITY JR., W.; YOUNG, W. IS-LM: an inquest. History of Political Economy, v. 27, n. 1, spring, p. 1-41, 1995.

FLASCHE, P.; REINER, F; SEMMLER, W. Dynamic macroeconomics. Cambridge: The MIT Press, 1997. 
HAHN, F; SOLOW, R. A critical essay on modern macroeconomic theory. Cambridge: The MIT Press, 1997.

JOHNSON, H. H. The general theory after twenty-five years. American Economic Review. Papers and Proceedings, v. LI, n. 2, p.1-17, 1961.

KEYNES, J. M. The general theory of employment, interest and money. London: Macmillan; C.W. VII., 1936.

KOOPMANS, T. C. Three essays on the state of economic science. Nova York: McGraw Hill, 1957.

MODIGLIANI, F. Liquidity preference and the theory of interest and money. Econometrica, v. 12, p. 45-88, Jan. 1944.

PONTI, G. Continuous-time selection dynamics - theory and practice. Research in Economics, v. 54, p. 187-214, 2002.

PRADO, E. F. S. Dilema dos prisioneiros e dinâmicas evolucionárias. Estudos Econômicos, v. 29, n. 2, p. 249-266, abr.-jun. 1999.

. Dois modelos clássicos de economia monetária. Economia Aplicada, v. 5, n. 3 , p. 547-567, jul.-set. 2001.

; KADOTA, D. K.; SOROMENHO, J. E. C. Survival of technologies: an evolutionary game approach. Economia Aplicada, v. 7, n. 2, jun. 2003.

SILVEIRA, J. J. Ciclos goodwinianos e o processo de concorrência num ambiente de racionalidade limitada. 2001. Tese (Doutorado) - IPE - FEA - USP.

. Ciclos clássicos num ambiente de racionalidade limitada. Estudos Econômicos, v. 33 n. 4, p. 701-734, out.-dez. 2003.

. Macrodinâmicas de crescimento em uma economia Solow-Swan com migração: uma abordagem de jogos evolucionários. Estudos Econômicos, v. 37, n. 2, p. 293-327, 2007.

; LIMA, G. T. Conhecimento imperfeito, custo de otimização e racionalidade limitada: uma dinâmica evolucionária de ajustamento nominal incompleto. In: XXXIV Encontro Nacional de Economia, Salvador, 2006.

SILVEIRA, J. J.; SANSON, J. R. The Harris-Todaro labor allocation mechanism as an evolutionary game. In: Latin American Meeting of the Econometric Society, Santiago, 2004.

SOROMENHO, J. E. C. Equilibrio e desemprego - um estudo sobre o pensamento keynesiano. 2003. Tese (Livre Docência) - FEA - USP, São Paulo.

; KADOTA, D. K.; PRADO, E. F. S. Scale and externalities in an evolutionary game model. Estudos Econômicos, v. 31, n. 3, p. 529-550, jul- set. 2001.

TOBIN, J. Price flexibility and the stability of full-employment equilibrium. In: BARKAI, H; FISCHER, S.; LIVIATAN, N. (Ed.). Monetary theory and thought: essays in honour of Don Patinkin. London: Macmillan, 1993. 
. Price flexibility and output stability. In: SEMMLER, W.. Business Cycles: theory and empirical methods. Boston: Kluwer Academic Publisher, 1994.

An overview of the general theory. In: HARCOURT, G. C.; RIACH, P. A. (Eds.). A 'second edition' of the general theory. London and New York: Routledge, 1997. v. 2, p. 3-27.

VEGA-REDONDO, F. Evolution, games and economic behaviour. Oxford: Oxford University Press, 1996.

WEIBULL, J. W. Evolutionary game theory. Cambridge: MIT Press, 1995.

\section{APÊNDICE}

Para provar a continuidade da função $\phi\left(\alpha, n_{1}, A\right)$ para pontos $\left(0, n_{1}, A\right)$ observe, inicialmente, que no subconjunto $\left\{\left(\alpha, n_{1}, A\right) \in \Omega_{1} \times R_{+}: \alpha \neq 0\right\}$, pelo teorema da função implícita, as derivadas

$$
\phi_{\alpha} \equiv \frac{\partial \omega_{2}^{*}}{\partial \alpha}=\frac{f\left(\frac{c n_{1}}{\alpha}\right)-\frac{c n_{1}}{\alpha} f^{\prime}\left(\frac{c n_{1}}{\alpha}\right)-f\left(g\left(\omega_{2}\right)\right)}{D_{1}-(1-\alpha) \omega_{2} g^{\prime}\left(\omega_{2}\right)}
$$

$\mathrm{e}$

$$
\phi_{n_{1}} \equiv \frac{\partial \omega_{2}^{*}}{\partial n_{1}}=\frac{c f^{\prime}\left(\frac{c n_{1}}{\alpha}\right)}{D_{1}-(1-\alpha) \omega_{2} g^{\prime}\left(\omega_{2}\right)}
$$

são contínuas, e, portanto, limitadas. No subconjunto $\left\{\left(\alpha, n_{1}, A\right) \in \Omega_{1} \times R_{+}\right.$: : $\alpha=0\}$ as derivadas laterais $\phi_{n_{1}}$ e $\phi_{\alpha}$ não são definidas. Não obstante, vale a seguinte propriedade:

Lema 7.1. $\phi_{\alpha}$ e $\phi_{n_{1}}$ são limitadas quando $x_{1}^{s}=\left(\alpha^{s}, n_{1}^{s}, A^{s}\right) \in \Omega_{1} \times R_{+}$tende a $\left(0, n_{1}, A\right)$ para $s \rightarrow \infty$.

Prova: O denominador $\partial E / \partial \omega_{2}=D_{1}-(1-\alpha) \omega_{2} g^{\prime}\left(\omega_{2}\right)$, comum a ambas as derivadas, é estritamente positivo. O numerador de $\phi_{\alpha}$ é $f\left(\frac{c n_{1}}{\alpha}\right)-\frac{c n_{1}}{\alpha} f^{\prime}\left(\frac{c n_{1}}{\alpha}\right)-$ $-f\left(g\left(\omega_{2}^{*}\right)\right)$. Como $f(0)=0$ e supf $=b, f\left(g\left(\omega_{2}^{*}\right)\right)$ é limitada. Para examinar a expressão $f\left(\frac{c n_{1}}{\alpha}\right)-\frac{c n_{1}}{\alpha} f^{\prime}\left(\frac{c n_{1}}{\alpha}\right)$, considere $v=\frac{c n_{1}}{\alpha}$. Sabemos que $f(v) \leq b$ e $v f^{\prime}(v) \geq 0$. Logo, $f(v)-v f^{\prime}(v) \leq b$. Ademais, $f(v)-v f^{\prime}(v) \geq 0$ sempre que 
$f(v) / v \geq f^{\prime}(v)$, ou seja, no caso em que a produtividade média é maior ou igual à produtividade marginal, o que sempre se verifica sob a hipótese de rendimentos marginais decrescentes. Portanto, $0 \leq f(v)-v f^{\prime}(v) \leq b$. Logo, para qualquer seqüência $x_{1}^{s}$ que tenda a $\left(0, n_{1}, A\right)$ quando $s \rightarrow \infty$, existe uma vizinhança em torno de $\left(0, n_{1}, A\right)$ na qual $f(v)-v f^{\prime}(v) \in[0, b]$. Segue-se que o numerador de $\phi_{\alpha}$ também é limitado e, portanto, existe $M>0$ tal que $\lim _{s \rightarrow \infty} \mid \phi_{\alpha}$ $\left(\alpha^{s}, n_{1}^{s}, A^{s}\right) \mid \leq M$ sempre que $\lim _{s \rightarrow \infty}\left(\alpha^{s}, n_{1}^{s}, A^{s}\right)=\left(0, n_{1}, A\right)$.

Para $\phi_{n_{1}}$, o numerador é $c f^{\prime}\left(c n_{1} / \alpha\right)$. Como, por hipótese, a função de produtividade marginal é limitada, existe $M>0$ finito tal que $\lim _{s \rightarrow \infty}\left|\phi_{n_{1}}\left(\alpha^{s}, n_{1}^{s}, A^{s}\right)\right| \leq M$ sempre que $\lim _{s \rightarrow \infty}\left(\alpha^{s}, n_{1}^{s}, A^{s}\right)=\left(0, n_{1}, A\right)$.

Proposição 7.1. $\phi\left(\alpha, n_{1}, A\right)$ é contínua nos pontos $\left(0, n_{1}, A\right) \in \Omega_{1} \times R_{+}$.

Prova: Sejam $x=\left(0, n_{1}, A\right) \in \Omega_{1} \times R_{+} \quad$ e $\quad k=\left(k_{1}, k_{2}, k_{3}\right) \in R^{3} \quad$ tal que $x+k=\left(k_{1}, n_{1}+k_{2}, A+k_{3}\right) \in \Omega_{1} \times R_{+}$. Observe que $\phi(x)=\phi\left(0, n_{1}, A\right)$ e $\phi(x+k)=\phi\left(k_{1}, n_{1}+k_{2}, A+k_{3}\right)$. A variação da função $\phi\left(\alpha, n_{1}, A\right)$ na direção dada pelo vetor $k$ é $k_{0} \equiv \phi(x+k)-\phi(x)$. Ademais, $E(\phi(x), x)=E(\phi(x+k)$, $x+k)=0$. Segue-se que:

$$
E(\phi(x), x)-E(\phi(x+k), x+k)=0
$$

Tomemos um escalar $\tau \in[0,1] \subset R$ e construamos a seguinte função paramétrica:

$$
\digamma(\tau)=E\left(\phi(x)+k_{0} \tau, x+k \tau\right)
$$

Diferenciando em relação a $\tau$, obtemos para qualquer $\tau \in(0,1]$ :

$$
\digamma^{\prime}(\tau)=\frac{\partial E}{\partial \omega_{2}} k_{0}+\frac{\partial E}{\partial \alpha} k_{1}+\frac{\partial E}{\partial n_{1}} k_{2}+\frac{\partial E}{\partial A} k_{3}=\nabla E\left(\phi(x)+k_{0} \tau, x+k \tau\right) \cdot \ell
$$


onde $\nabla E(\cdot)$ é o vetor gradiente da função de excesso de demanda e $\ell=\left(k_{0}, k_{1}, k_{2}, k_{3}\right)$. Observe que o vetor gradiente não está definido para $\tau=0$. Sabemos que a função de excesso de demanda $E(\phi(x), x)$ é contínua no domínio convexo $(0, a] \times \Omega_{1} \times R_{+}$. A função paramétrica $\digamma(\tau)$ é, portanto, contínua para qualquer $\tau \in[0,1]$. Em particular, $\digamma(\cdot)$ é contínua no subintervalo $[0, \tau] \subset[0,1] \mathrm{e}$ derivável em qualquer ponto interior deste subintervalo. Existe, então, pelo teorema do valor médio de Lagrange, uma constante $\theta \in(0,1)$ tal que:

$$
\frac{\digamma(\tau)-\digamma(0)}{\tau}=\digamma^{\prime}(\theta \tau)
$$

A partir de (7.5) e (7.6), temos:

$$
\frac{\digamma(\tau)-\digamma(0)}{\tau}=\nabla E\left(\phi(x)+k_{0} \theta \tau, x+k \theta \tau\right) \cdot \ell
$$

Fazendo $\tau=1$, obtemos:

$$
\digamma(1)-\digamma(0)=\nabla E\left(\phi(x)+k_{0} \theta \tau, x+k \theta \tau\right) \cdot \ell
$$

Com base em (7.3) e (7.4), inferimos:

$$
\digamma(1)-\digamma(0)=E\left(\phi(x)+k_{0}, x+k\right)-E(\phi(x), x)=0
$$

Logo, de (7.8) e (7.9):

$$
\nabla E\left(\phi(x)+k_{0} \theta \tau, x+k \theta \tau\right) \cdot \ell=0
$$

Considerando (7.5), ao dividirmos ambos os lados de (7.10) por $\partial E / \partial \omega_{2}=D_{1}-$ $-(1-\alpha) \omega_{2} g^{\prime}\left(\omega_{2}\right)>0$ e isolarmos $k_{0} \equiv \phi(x+k)-\phi(x)$, chegamos a:

$$
\phi(x+k)-\phi(x) \equiv k_{0}=\phi_{\alpha} k_{1}+\phi_{n_{1}} k_{2}+\phi_{A} k_{3}
$$

onde as derivadas $\phi_{\alpha}=-\frac{\partial E / \partial \alpha}{\partial E / \partial \omega_{2}}, \phi_{n_{1}}=-\frac{\partial E / \partial n_{1}}{\partial E / \partial \omega_{2}}$ e $\phi_{A}=-\frac{\partial E / \partial A}{\partial E / \partial \omega_{2}}$ são calculadas no ponto $\left(\phi(x)+k_{0} \theta, x+k \theta\right)$

Por fim, observe que: 


$$
\lim _{k \rightarrow(0,0,0)} k_{0}=\lim _{k \rightarrow(0,0,0)} \phi_{\alpha} k_{1}+\phi_{n_{1}} k_{2}+\phi_{A} k_{3}=0
$$

pois $\phi_{A}$ é contínua em todo o domínio (inclusive para $\alpha=0$ ) e, portanto, limitada; além disso, como demonstrado no lema, $\phi_{\alpha}$ e $\phi_{n 1}$ são contínuas para $\alpha \neq 0$ e limitadas para $\alpha$ tendendo a zero. Então,

$$
\lim _{k \rightarrow(0,0,0)} \phi(x+k)=\phi(x)
$$

o que prova que $\phi\left(\alpha, n_{1}, A\right)$ também é contínua em $\alpha=0$. 